\title{
Energy Near-Optimal Control Strategies for Industrial and Traction Drives with a.c. Motors
}

\author{
Ján Vittek, ${ }^{1}$ Peter Butko, ${ }^{1}$ Branislav Ftorek, ${ }^{2}$ Pavol Makyš, ${ }^{1}$ and Lukáš Gorel ${ }^{1}$ \\ ${ }^{1}$ Faculty of Electrical Engineering, University of Žilina, Žilina, Slovakia \\ ${ }^{2}$ Faculty of Mechanical Engineering, University of Žilina, Žilina, Slovakia \\ Correspondence should be addressed to Ján Vittek; jan.vittek@fel.uniza.sk
}

Received 11 October 2016; Revised 18 November 2016; Accepted 24 November 2016; Published 24 January 2017

Academic Editor: Mohammed Nouari

Copyright (C) 2017 Ján Vittek et al. This is an open access article distributed under the Creative Commons Attribution License, which permits unrestricted use, distribution, and reproduction in any medium, provided the original work is properly cited.

\begin{abstract}
The main contribution of this paper is a new rest-to-rest position control system for use with electric drives employing a.c. motors that is near-optimal with respect to combined electrical and frictional energy waste minimization. The friction has constant, linear, and quadratic components with respect to the rotor speed. The closeness to optimality is assessed by simulation, comparing the energy loss of the new control system with that predicted by computed optimal controls. The application of the near-optimal control system is rendered straightforward by using a symmetrical trapezoidal speed-time profile. This is provided by an energy saving reference position generator whose output is faithfully followed by means of a feedback control law based on forced dynamics control yielding prescribed closed loop dynamics, together with a matched zero dynamic lag precompensator. For load torque consisting of constant, linear, and quadratic components also maneuver time is optimized if it can be chosen arbitrary. Two case studies, one applied to position control of rotational drive and second one applied to train movement, confirm the possibilities of achieving energy savings.
\end{abstract}

\section{Introduction}

As a result of environmental concerns and minimization of energy consumption the calculation of energy optimal reference trajectories for electric drives is significant for possible energy saving [1]. The paper presents applications of energy optimal control theory based on Euler-Lagrange minimization to the rotational movement of industrial drives and to the traction motor of railway vehicle. Both applications assume load which consists of constant, linear, and quadratic components with respect to the rotor speed.

Two generally valid position control strategies respecting electrical losses of motor as well as mechanical losses minimization are developed and verified for comparison of their energy demands. The first strategy is based strictly on mathematical, Euler-Lagrange approach [2] while the second one is based on loss model exploiting symmetrical trapezoidal speed profile for electrical and mechanical losses minimization. The second approach is characterized with truly finite settling time and for prescribed movement allows precise prediction of all components of consumed energy.
Total consumed energy of both control strategies is evaluated via time integral of the input power. For comparison of possible energy savings of both control strategies the investigations are completed with position control based on modified trapezoidal speed profile and control strategy based on triangular speed profile.

Principles of vector control and forced dynamics control are used for energy demands evaluation of all presented control strategies. Vector control of a.c. drives as a novel control strategy had opened possibility of controlling torque and field components of stator current independently and brought wide potential for considerable reduction of drive losses [3]. Significant energy savings also contributed to rapid spread of variable speed drives in industry and transportation. For simulations of energy saving position control strategies a new "forced dynamics control" technique (FDC) of electric drives employing a.c. motors based on feedback linearization [4] and enabling realization of various prescribed dynamic responses to speed demands is exploited.

The individual approaches to energy-efficient position control while respecting prescribed maneuver time between 
stops differ significantly. The problems of optimal trajectory planning based on the nonlinear continuous-time model and continuous state-space model of train movement are well presented in overview of Wang et al. [5]. The methods of solutions can be characterized as analytical solution and numerical optimization. The analytical methods have difficulties in finding general solutions due to complex nonlinear terms and consideration of the constraints. For the numerical optimization approaches the optimal solution is not always guaranteed too. The possible outcome is to solve the optimal trajectory planning problem as a mixed integer linear programming problem, which can be solved efficiently exploiting available solvers.

Combination of Model Predictive Control (MPC) and differential flatness is used by Pham et al. [6] for efficient energy management of an elevator employing PMSM supplied from microgrid (solar panel and supercapacitor battery, backed-up with three-phase net). The flatness formulation of the considered system profiles consisting of stator currents and rotor speed are generated to minimize the stator resistances energy dissipation. Then, MPC is used to track given profiles while satisfying state input constraints. Simulation results using real system numerical data compare the energy efficiency of the designed method with method which combines the Maximum Torque per Ampere (MTPA) principles in connection with the trapezoidal speed profile.

The drive's loss model is used by Blank et al. [7] for optimal trajectory planning. Iterative algorithm, fmincon, finds a constrained minimum of a scalar function of several variables to compute prescribed acceleration. The resulting trajectories (constant for time-minimal interval, constant for half of maneuver time, sinusoidal, and linearly monotonously falling) respect also prescribed jerk and reduce copper losses as well as losses of power electronics.

Minimization of the total dissipated energy taking into account Coulomb and viscous friction has been proposed by Xuejun et al. [8] for the drive employing d.c. motor with reduction gear. The optimal drive velocity and current functions are obtained as a function of the optimal zero crossing time when input torque of gear is changed from positive to negative. Comparisons of the dc motor drive consumption for optimized control algorithm and trapezoidal velocity function have revealed energy savings of optimized control algorithm, which were proportional to a moment of inertia.

For minimization of dissipated energy of battery powered mobile wheeled robot, C. H. Kim and B. K. Kim [9] analyzed and implemented three-step velocity control method. Two various forms of acceleration and deceleration profiles (constant and exponential) are investigated to find minimal energy consumption. As energy near-optimal solution the analysis reveals exponential acceleration profile completed with constant cruising speed and linear deceleration (step acceleration-cruising speed-trapezoidal breaking). Objective function which respects drive's energy is developed for threestep velocity control. Solution in real time finds efficient binary search algorithm. Suggested three-step velocity control extends the working time of mobile robot up to $30 \%$.
A single-axis trajectory generator for point-to-point motion control was proposed by Thirachai et al. [10]. Generated profiles correspond to trapezoidal velocity function that can modify system parameters such as the destination, speed, and acceleration. A dead-beat controller is exploited when the current state variables are approaching the equilibrium point. Simulation results confirm that proposed system can provide finite steps and a zero steady-state error and showed that the overall performance is similar to a conventional method.

Direct robust adaptive controller for improvement of the tracking performance of high-speed train was developed by Luo and $\mathrm{Xu}$ [11]. The controller is designed using the back-stepping method to deal with nonlinearities and the parameterized uncertainties in the high-speed train dynamic model. Then, the projection algorithm is used to improve the robustness of the proposed control algorithm for slowly timevarying parameter. Theoretical analysis and the simulation results confirmed that the proposed algorithm can guarantee the stability of the designed closed loop system with the tracking error converging to a residual set exponentially.

For road vehicle employing PMSM Lu et al. in [12] solve problem of global energy optimization to obtain the optimal velocity reference. Dynamic programming taking into account efficiency model of the drive system and complex road conditions determines trajectory with minimum energy consumption. For control of speed of hybrid electric vehicle by controlling the throttle position Yadav et al. study performances of various controller (five various controllers) in [13]. Comparative analysis confirmed optimal performance of linear-quadratic regulator (LQR) for optimization of vehicle drive train efficiency.

To minimize energy expenditures of suburban trains De Martinis and Gallo [14] proposed new driving strategies using a railway simulation model as a subroutine. Their approach results in the definition of two optimized train speed profiles. The first model is based on energy saving approach while the second one is based on energy recovery strategies. Two scenarios for resulting speed profiles (with coasting and without) were proposed and tested on a real-scale case involving a suburban line for both models. Simulation results show a promising reduction in energy consumption with the optimized energy-efficient speed profile for a prescribed running time between two stops.

Sheepmaker et al. [15] studied optimal control of the train with respect to braking (mechanical, mechanical combined with regenerative, and regenerative). Pseudo-spectral method is used to solve energy-efficient train control, which is modelled as an optimal control problem over distance. Results of analysis have shown that under regenerative breaking the optimal cruising speed is lower than without, the coasting regime is shorter, and the braking regime starts earlier. This led to extra energy saving what emphasizes the importance of kinetic energy utilization. The conclusion stresses substantial influence of regenerative braking on energy-efficient driving strategy and lower energy consumption. 
Different control mechanism for a diesel-electric locomotive was used by Albrecht et al. [16] to design speedholding control strategy for the train movement between two stations with nonconstant gradient and prescribed travelling time. Energy optimal type strategy based on discrete throttle settings alternates phases of coasting and maximum power to approximate the ideal minimum consumption strategy. The method has been successfully implemented in connection with special computation device for calculation of the optimal switching points and to provide in-cab advice to train drivers.

Detailed program for traction and brake applications capable of energy consumption minimization in the moving train along a given route for a given travel time was developed by Khmelnitsky [17]. The maximum principle is used to obtain the analytical information about optimal operation regimes and their sequences respecting also mixed and state constraints. Numerical algorithm exploiting this information then finds the optimal velocity profile. Designed control algorithm has capability of arbitrary restart due to outside conditions change and then recalculates the optimal velocity profile by constructing a new optimal profile for the remaining route length and travel time using the current speed as the initial one. Numerical examples confirm inherent accuracy and fast response of algorithm based on its analytical origin.

Contribution of this paper is the development and application of two energy near-optimal control strategies for the drives with rotational or translation movement and comparisons of their energy demands with modified strategies used for position control. To ensure the same conditions of comparison the regenerative braking is assumed for all presented strategies.

Designed control structure for simulation of energy saving control is shown in Figure 1 and consists of "Energy Optimal or Energy Near-Optimal Profile Generator" which is capable, from demanded position, $\theta_{r d}$; prescribed maneuver time, $T_{m}$; and computed load torque, $\Gamma_{L}$, of producing reference inputs: acceleration, $\varepsilon_{r i}$; speed, $\omega_{r i}$; and position, $\theta_{r i}$ for drive's position control system. Block "Load Torque Computation" evaluates value of load torque as a function of drive's speed using its individual coefficients for constant, $A$; viscous, $B$; and quadratic, $C$ components (predetermined offline). The task of precompensator is to form control signal, $\theta_{r d}^{\prime}$, to ensure better tracking abilities of prescribed state variables. Design of precompensator is described in detail in Section 3.3 for vector controlled drive combined with FDC [18].

Position control system itself has nested control structure consisting of outer position control loop with proportional regulator and standard inner speed control loop for a.c. motors (IM or PMSM) with PI speed regulator. Speed control loop respects vector control principles in chosen reference frame [19]. Motor supply voltages, $u_{d}$ and $u_{q}$, are produced by PI regulators in direct and quadrature axis of the vector controlled drive.

For real time implementation the circuitry shown in Figure 1 is completed with standard transformation blocks (from system coupled with chosen reference frame into rotational twophase orthogonal system and from two-phase into three-phase system) and inverter with space vector modulation.

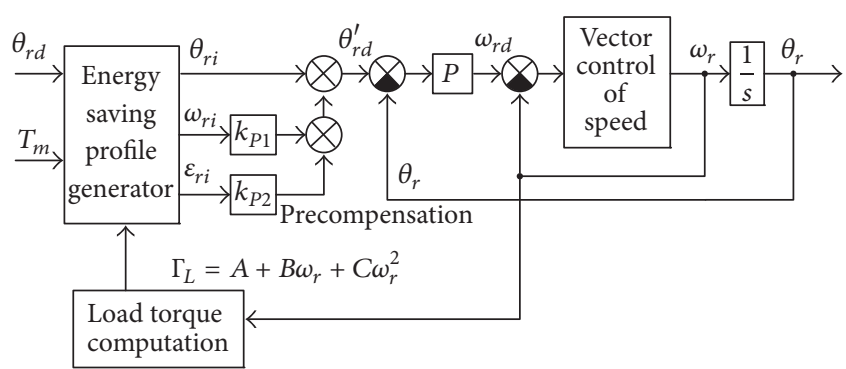

FIGURE 1: Blok diagram of the energy saving position control system.

\section{Theoretical Background}

State-space notation, $\dot{\mathbf{x}}=\mathbf{A} \mathbf{x}+\mathbf{B u}$ and $y=\mathbf{C}^{\mathrm{T}} \mathbf{x}$, is convenient for a.c. motors description as well as for solving energy optimization task for the drive dynamics [20]. IM state model consisting of stator current vector, I; rotor flux vector, $\Psi$; rotor speed, $\omega_{r}$; and rotor position, $\theta_{r}$, formulated in the coordinate system rotating in arbitrary speed, $\omega_{k}$, is

$$
\begin{aligned}
\dot{\mathbf{I}} & =c_{1}\left[\mathbf{U}-a_{1} \mathbf{I}+c_{2} \mathbf{P}\left(\omega_{r}\right) \Psi\right]+p \omega_{k} \mathbf{T}^{\mathrm{T}} \mathbf{I}, \\
\dot{\Psi} & =c_{4} \mathbf{I}-\mathbf{P}\left(\omega_{r}\right) \Psi+p \omega_{k} \mathbf{T}^{\mathrm{T}} \Psi \\
\dot{\omega}_{r} & =\frac{1}{J_{r}}\left(\Gamma_{\mathrm{el}}-\Gamma_{L}\right)=\frac{1}{J_{r}}\left(c_{5} \Psi^{\mathrm{T}} \mathbf{T}^{\mathrm{T}} \mathbf{I}-\Gamma_{L}\right), \\
\dot{\theta}_{r} & =\omega_{r},
\end{aligned}
$$

where $\mathbf{I}^{\mathrm{T}}=\left[\begin{array}{ll}l_{d} & \iota_{q}\end{array}\right]$ is the stator current, $\mathbf{U}^{\mathrm{T}}=\left[\begin{array}{ll}u_{d} & u_{q}\end{array}\right]$ is the stator voltage, $\Psi^{\mathrm{T}}=\left[\begin{array}{ll}\Psi_{D} & \Psi_{\mathrm{Q}}\end{array}\right]$ is the rotor magnetic flux, $\gamma_{e}$ is the torque developed by the motor, $\omega_{r}$ is the mechanical rotor speed, $\omega_{k}$ is the electrical angular speed valid for arbitrary coordinate system rotating at $\omega_{k}$, and $J_{r}$ is moment of inertia related to motor shaft. The constants are given as $c_{1}=L_{r} /\left(L_{s} L_{r}-L_{m}^{2}\right), c_{2}=L_{m} / L_{r}, c_{3}=R_{r} / L_{r}=1 / T_{r}$, $c_{4}=L_{m} / T_{r}, c_{5}=1.5 p L_{m} / L_{r}$, and $a_{1}=R_{s}+R_{r}\left(L_{m}^{2} / L_{r}^{2}\right)$, where $L_{s}, L_{r}$, and $L_{m}$ are the stator and rotor inductance and their mutual inductance, respectively. $R_{s}$ and $R_{r}$ are the stator and rotor resistances and $p$ is the number of pole pairs. Advantage of this notation is definition of motor resistance, $a_{1}$, which consists of stator resistance and rotor resistance related to the stator what enables us to evaluate copper losses of stator and rotor as a function of stator currents. Matrices $\mathbf{P}\left(\omega_{r}\right)$ and $\mathbf{T}$ are defined as

$$
\begin{aligned}
\mathbf{P}\left(\omega_{r}\right) & =\left[\begin{array}{cc}
c_{3} & p \omega_{r} \\
-p \omega_{r} & c_{3}
\end{array}\right], \\
\mathbf{T} & =\left[\begin{array}{cc}
0 & -1 \\
1 & 0
\end{array}\right] .
\end{aligned}
$$

In a similar way, the state model of PMSM consisting of vectors, stator current, I; stator flux, $\Psi$; rotor speed, $\omega_{r}$; 
and rotor position, $\theta_{r}$, formulated in the rotor-fixed $d_{-} q$ coordinate system has the following form:

$$
\begin{aligned}
\dot{\mathbf{I}} & =\mathbf{P}\left(\omega_{r}, R_{s}\right) \mathbf{I}-\frac{p \omega_{r}}{L_{q}} \boldsymbol{\Psi}_{\mathrm{PM}}+\mathbf{L}_{\mathbf{R}} \mathbf{U}, \\
\dot{\omega}_{r} & =\frac{1}{J_{r}}\left(\Gamma_{\mathrm{el}}-\Gamma_{L}\right)=\frac{1}{J_{r}}\left(c \boldsymbol{\Psi}^{\mathrm{T}} \mathbf{T}^{\mathrm{T}} \mathbf{I}-\Gamma_{L}\right), \\
\dot{\theta}_{r} & =\omega_{r},
\end{aligned}
$$

where $\Psi^{\mathrm{T}}=\left[\begin{array}{ll}\Psi_{d} & \Psi_{q}\end{array}\right]$ is the stator magnetic flux in which $\Psi_{d}=L_{d} i_{d}+\Psi_{\mathrm{PM}}$ and $\Psi_{q}=L_{q} i_{q}, \mathbf{I}^{\mathrm{T}}=\left[\begin{array}{ll}\iota_{d} & \iota_{q}\end{array}\right]$ is the stator current, $\mathbf{U}^{\mathrm{T}}=\left[\begin{array}{ll}u_{d} & u_{q}\end{array}\right]$ is the stator voltage, $\gamma_{e}$ is the motor torque, $\omega_{r}$ is the rotor speed, and $J_{r}$ is moment of inertia. Constant $c$ is given as $c=3 p / 2$ and $L_{d}, L_{q}$ are the stator inductances in direct and quadratic axis, respectively. $R_{s}$ is the stator resistance and $p$ is the number of pole pairs. Matrices $\mathbf{P}\left(\omega_{r}, R_{s}\right), \Psi_{\mathrm{PM}}$, and $\mathrm{L}_{\mathbf{R}}$ have the following form:

$$
\begin{aligned}
\mathbf{P}\left(\omega_{r}, R_{s}\right) & =\left[\begin{array}{cc}
-\frac{R_{s}}{L_{d}} & \frac{p \omega_{r} L_{q}}{L_{d}} \\
-\frac{p \omega_{r} L_{d}}{L_{q}} & -\frac{R_{s}}{L_{q}}
\end{array}\right], \\
\Psi_{\mathrm{PM}} & =\left[\begin{array}{c}
0 \\
\Psi_{\mathrm{PM}}
\end{array}\right], \\
\mathbf{L}_{\mathbf{R}} & =\left[\begin{array}{cc}
\frac{1}{L_{d}} & 0 \\
0 & \frac{1}{L_{q}}
\end{array}\right] .
\end{aligned}
$$

To derive generally valid conditions for energy optimal position control the system is described as torque generator respecting both a.c. motor (IM, SMPM) with three differential equations, for rotor position, $\theta_{r}$; speed, $\omega_{r}$; and electric torque, $\gamma_{e}$, which are completed with an equation for load torque: $\gamma_{L}=A+B \omega_{r}+C \omega_{r}^{2}$. Coefficients $A, B$, and $C$ respect Coulomb, viscous and quadratic friction, respectively. Load torque derivative has the form $\dot{\gamma}_{L}=\left(B+2 C \omega_{r}\right) \dot{\omega}_{r}$ and complete dynamical system is

$$
\begin{aligned}
& \begin{array}{l}
{\left[\begin{array}{l}
\dot{\theta}_{r} \\
\dot{\omega}_{r} \\
\dot{\gamma}_{e} \\
\dot{\gamma}_{L}
\end{array}\right]} \\
=\left[\begin{array}{cccc}
0 & 1 & 0 & 0 \\
0 & 0 & \frac{1}{J_{r}} & -\frac{1}{J_{r}} \\
0 & -k_{\omega} & -k_{\gamma} & 0 \\
0 & 0 & \frac{\left(B+2 C \omega_{r}\right)}{J_{r}} & -\frac{\left(B+2 C \omega_{r}\right)}{J_{r}}
\end{array}\right]\left[\begin{array}{l}
\theta_{r} \\
\omega_{r} \\
\gamma_{e} \\
\gamma_{L}
\end{array}\right]
\end{array} \\
& \begin{array}{l}
{\left[\begin{array}{l}
\dot{\theta}_{r} \\
\dot{\omega}_{r} \\
\dot{\gamma}_{e} \\
\dot{\gamma}_{L}
\end{array}\right]} \\
=\left[\begin{array}{cccc}
0 & 1 & 0 & 0 \\
0 & 0 & \frac{1}{J_{r}} & -\frac{1}{J_{r}} \\
0 & -k_{\omega} & -k_{\gamma} & 0 \\
0 & 0 & \frac{\left(B+2 C \omega_{r}\right)}{J_{r}} & -\frac{\left(B+2 C \omega_{r}\right)}{J_{r}}
\end{array}\right]\left[\begin{array}{l}
\theta_{r} \\
\omega_{r} \\
\gamma_{e} \\
\gamma_{L}
\end{array}\right]
\end{array} \\
& +\left[\begin{array}{c}
0 \\
0 \\
k_{U} \\
0
\end{array}\right] u_{q},
\end{aligned}
$$

where $J_{r}$ is moment of inertia related to rotor and for PMSM constants are defined as $k_{\omega}=p k_{t} \Psi_{\mathrm{PM}}, k_{\gamma}=R_{s} / L_{q}$, and $k_{U}=$ $k_{t} / L_{q}$. PMSM torque constant is defined as $k_{t}=c \Psi_{\mathrm{PM}}$. For IM these constants are $k_{\omega}=k_{t}\left(c 2-\sigma L_{s} / L_{m}\right) p \Psi_{D}, k_{\gamma}=a_{1} / \sigma L_{1}$, and $k_{U}=k_{t} / \sigma L_{s}$. IM torque constant is defined as $k_{T}=$ $c_{5} \psi_{D}$ and $u_{q}$ is $q_{-}$axis excitation voltage. For simplification of further calculations a new constant defined as $k_{m}=k_{t}^{2} / 2 R$ (for PMSM $R=R_{s}$ and for IM $R=a_{1}$ ) was introduced.

2.1. Energy Optimal Control. Mathematical approach based on Euler-Lagrange minimization is exploited to derive conditions for energy optimal control. Functional for energy expenses minimization covering electrical-copper and mechanical-friction losses during maneuver interval, $T_{m}$, is defined as

$$
I_{T}=\frac{R}{k_{T}^{2}} \int_{0}^{T_{m}} \gamma_{e}^{2} d t+\int_{0}^{T_{m}} \gamma_{L} \omega_{r} d t
$$

Overall cost function, $I_{P}$, for drive's losses minimization consists of minimization functional (11) completed with description of the state variables, to which multiplication with Lagrange coefficients, $\lambda_{i}$ for $i=1,2,3$, is added:

$$
\begin{aligned}
I_{P}= & \frac{R}{k_{T}^{2}} \gamma_{e}^{2}+\gamma_{L} \omega_{r}+\lambda_{1}\left(J_{r} \dot{\omega}_{r}-\gamma_{e}+\gamma_{L}\right) \\
& +\lambda_{2}\left(\dot{\theta}_{r}-\omega_{r}\right)+\lambda_{3}\left(\dot{\gamma}_{L}-B \dot{\omega}_{r}-2 C \omega_{r} \dot{\omega}_{r}\right) .
\end{aligned}
$$

The solution of minimization problem requires calculation of Euler-Lagrange equations, found via partial derivatives of the designed cost function, $I_{P}$, for given state variables as

$$
\frac{\partial I_{P}}{\partial x_{i}}=\frac{d}{d t}\left(\frac{\partial I_{P}}{\partial \dot{x}_{i}}\right) \text { for } x_{i}=\theta_{r}, \omega_{r}, \gamma_{e}, \gamma_{L}
$$

resulting in

$$
\begin{gathered}
\lambda_{1}=\frac{2 R}{k_{t}^{2}} \gamma_{e}, \\
\dot{\lambda}_{3}=\omega_{r}+\lambda_{1} \\
\dot{\lambda}_{2}=0, \quad \text { for } x_{i}=\gamma_{e}, \gamma_{L}, \\
J_{r} \dot{\lambda}_{1}-B \dot{\lambda}_{3}-2 C \omega_{r} \dot{\lambda}_{3}=\gamma_{L}-\lambda_{2} \\
\quad \text { for } x_{i}=\theta_{r}, \omega_{r} .
\end{gathered}
$$


After manipulation of variables the matching conditions given by individual Euler-Lagrange equations can be summarized into the highly nonlinear system written in matrix form as

$$
\begin{aligned}
{\left[\begin{array}{c}
\dot{\lambda}_{1} \\
\dot{\lambda}_{2} \\
\dot{\omega}_{r} \\
\dot{\theta}_{r}
\end{array}\right]=} & {\left[\begin{array}{cccc}
\frac{B}{J_{r}} & -\frac{1}{J_{r}} & \frac{2 B}{J_{r}} & 0 \\
0 & 0 & 0 & 0 \\
\frac{k_{m}}{J_{r}} & 0 & -\frac{B}{J_{r}} & 0 \\
0 & 0 & 1 & 0
\end{array}\right]\left[\begin{array}{c}
\lambda_{1} \\
\lambda_{2} \\
\omega_{r} \\
\theta_{r}
\end{array}\right]+\left[\begin{array}{c}
\frac{1}{J_{r}} \\
0 \\
-\frac{1}{J_{r}} \\
0
\end{array}\right] A } \\
& +\left[\begin{array}{c}
\frac{2 C \lambda_{1}}{J_{r}} \\
0 \\
0 \\
0
\end{array}\right] \omega_{r}+\left[\begin{array}{c}
\frac{3 C}{J_{r}} \\
0 \\
C \\
-\frac{C}{J_{r}} \\
0
\end{array}\right] \omega_{r}^{2} .
\end{aligned}
$$

Nonlinear system (15) has to satisfy boundary conditions:

$$
\begin{array}{cl}
\text { for } t=0 & \omega_{r}=0, \\
& \theta_{r}=0, \\
\text { for } t=T_{m} & \omega_{r}=0, \\
& \theta_{r}=\theta_{r \mathrm{dem}} .
\end{array}
$$

Resulting nonlinear dynamical system (15) can be solved with only the iterative way. Numerical solutions of this system offer MATLAB function bvp4c.m (boundary value problem), which integrates system of ordinary differential equations subject to general two-point boundary conditions. Results of integration are profiles of acceleration, speed, and position satisfying Euler-Lagrange minimization approach for minimum energy requirements.

Analytical solution of (15) can be found for constant and linear components of load torque $(A, B)$ while assuming that quadratic component $C=0$. In this case the system is transformed into linear one:

$$
\left[\begin{array}{l}
\dot{\lambda}_{1} \\
\dot{\lambda}_{2} \\
\dot{\omega}_{r} \\
\dot{\theta}_{r}
\end{array}\right]=\left[\begin{array}{cccc}
\frac{B}{J_{r}} & -\frac{1}{J_{r}} & \frac{2 B}{J_{r}} & 0 \\
0 & 0 & 0 & 0 \\
\frac{k_{m}}{J_{r}} & 0 & -\frac{B}{J_{r}} & 0 \\
0 & 0 & 1 & 0
\end{array}\right]\left[\begin{array}{c}
\lambda_{1} \\
\lambda_{2} \\
\omega_{r} \\
\theta_{r}
\end{array}\right]+\left[\begin{array}{c}
\frac{1}{J_{r}} \\
0 \\
-\frac{1}{J_{r}} \\
0
\end{array}\right] A,
$$

with two real multiple and two complex conjugated eigenvalues located in

$$
\begin{aligned}
& l_{1,2}=0, \\
& l_{3,4}=\mp \frac{B}{J_{r}} \sqrt{1+\frac{k_{t}^{2}}{R_{s} B}} .
\end{aligned}
$$

General solution of the system consisting of homogenous and particular part has the following form:

$$
\begin{aligned}
& {\left[\begin{array}{l}
\lambda_{1} \\
\lambda_{2} \\
\omega_{r} \\
\theta_{r}
\end{array}\right]=C_{1} e^{-\left(\left(B / J_{r}\right) \sqrt{1+k_{t}^{2} / R_{s} B}\right) t}\left[\begin{array}{c}
\frac{2 R_{s} J_{r}}{k_{t}^{2}}\left[\frac{B}{J_{r}} \sqrt{\frac{B}{J_{r}}+\frac{k_{t}^{2}}{R_{s} J_{r}}}\left(-\sqrt{\frac{B}{J_{r}}}+\sqrt{\frac{B}{J_{r}}+\frac{k_{t}^{2}}{R_{s} J_{r}}}\right)\right] \\
0 \\
-\sqrt{\frac{B}{J_{r}} \sqrt{\frac{B}{J_{r}}+\frac{k_{t}^{2}}{R_{s} J_{r}}}} \\
1
\end{array}\right]}
\end{aligned}
$$

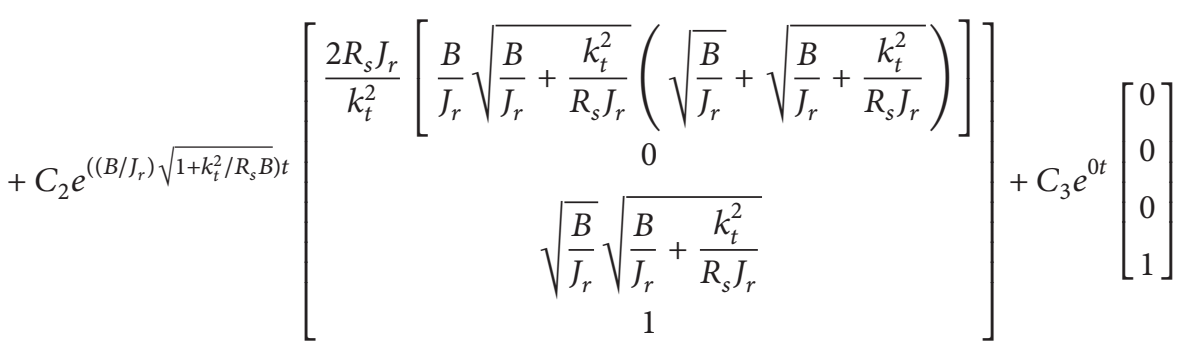

$$
\begin{aligned}
& +C_{4} e^{0 t}\left[\begin{array}{c}
\frac{2 B R_{s}}{k_{t}^{2}} \\
2 B\left(\frac{B R_{s}}{k_{t}^{2}}+1\right) \\
1 \\
1
\end{array}\right]+\left[\begin{array}{c}
\frac{2 R_{s}}{k_{t}^{2}}(A+B) \\
A+2 B+\frac{2 B R_{s} J_{r}}{k_{t}^{2}}(A+B) \\
1 \\
1
\end{array}\right] .
\end{aligned}
$$


The coefficient $c_{1-4}$ of (19) can be determined as solution of the following linear system:

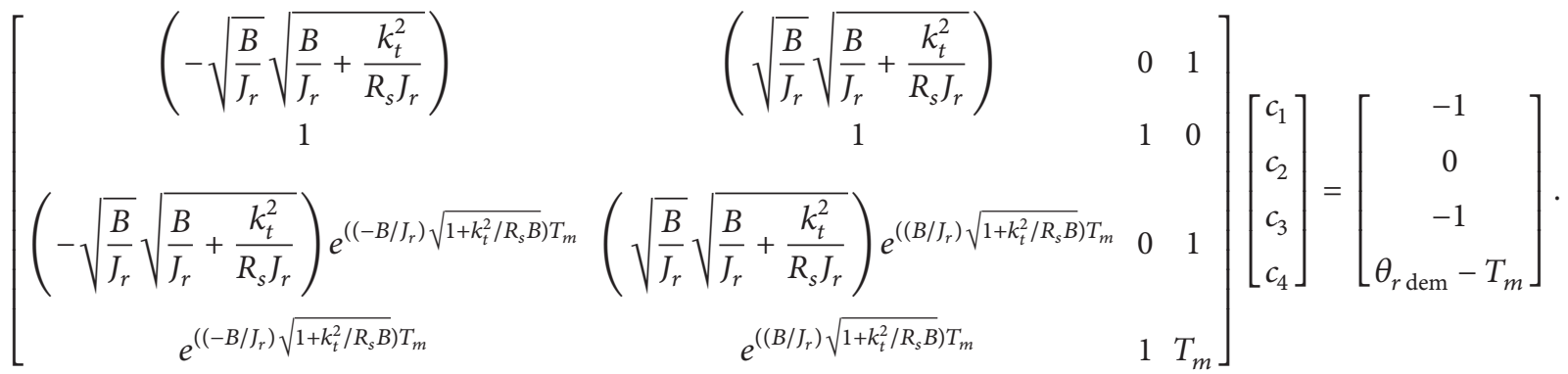

Energy optimal control strategy and resulting state variables were derived for torque generator generally valid conditions and therefore can be used for energy optimal control of the drives with arbitrary electric motor.

2.2. Modified Energy Near-Optimal Control Strategy. The second approach of modified energy near-optimal position control exploits symmetrical trapezoidal function for rotor speed together with related position profile approximating energy optimal variables quite closely. This strategy allows reaching demanded position via control on constant torque during acceleration and deceleration intervals and control on constant cruising speed in the middle interval. This approach was verified via detailed analysis respecting electrical losses in motor windings and friction losses together. Complete results for analysis of constant, linear, and quadratic dependent load torque based on this approach was described in [21]; therefore, the following is a brief description only.

Demanded rotor position, $\theta_{r d}$, and cruising speed magnitude, $\omega_{p}$, as a function of acceleration, $\varepsilon_{p}$, and its duration, $T_{\mathcal{E}}$, are for trapezoidal speed profile defined as

$$
\begin{aligned}
& \theta_{r d}=2 \int_{0}^{T_{\varepsilon}} \varepsilon_{p} t d t+\int_{0}^{T_{m}-2 T_{\varepsilon}} \omega_{p} d t=\varepsilon_{p}\left(T_{m} T_{\varepsilon}-T_{\varepsilon}^{2}\right), \\
& \omega_{p}=\int_{0}^{T_{\varepsilon}} \varepsilon_{p} d t=\varepsilon_{p} T_{\varepsilon} .
\end{aligned}
$$

Time of acceleration and deceleration, $T_{\varepsilon}$, under assumption of minimum value of maneuver time, $T_{m}$, and constant cruising speed, $\omega_{p}$, have the following relations:

$$
\begin{aligned}
& T_{\varepsilon}=\frac{T_{m}}{2}\left[1-\sqrt{1-\frac{4 \theta_{r d}}{\varepsilon_{p} T_{m}^{2}}}\right], \\
& T_{m} \geq 2 \sqrt{\frac{\theta_{r d}}{\varepsilon_{p}}}, \\
& \omega_{p}=\frac{T_{m}}{2}\left[\varepsilon_{p}-\sqrt{\varepsilon_{p}^{2}-\frac{4 \theta_{r d} \varepsilon_{p}}{T_{m}^{2}}}\right] .
\end{aligned}
$$

The generation of required profiles respecting losses minimization is based on analysis of friction and copper losses of the drive in steady-state (current dynamics due to inductances, $L_{d}, L_{q}$ of SMPM, and $L_{s}, L_{r}$ of IM and iron losses are neglected) as it is shown in Figure 2.

For composed friction torque, $\Gamma_{L}=A+B \omega+C \omega^{2}$, description of predictable current is divided into three time intervals as

$$
\begin{aligned}
& i_{q 1}(t)=I_{q d}+i_{q L}(t), \\
& i_{q 2}(t)=I_{q L}, \\
& i_{q 3}(t)=-I_{q d}+i_{q L}(t),
\end{aligned}
$$

where currents, $I_{q d}$ and $I_{q L}$, are constant: $I_{q d}=\varepsilon_{p}\left(J_{r} / c \Psi\right)$ and $I_{q L}=\Gamma_{L}\left(T_{0}\right) / c \Psi=A / c \Psi+B \omega_{p} / c \Psi+C \omega_{p}^{2} / c \Psi$, and current, $i_{q L}$, is time varying as $i_{q L}=\Gamma_{L}(t) / c \Psi=A / c \Psi+B \omega / c \Psi+C \omega^{2} / c \Psi$. Total energy to cover drive's losses, $W_{T}$, is expressed as a sum of energy to cover electrical and mechanical losses:

$$
W_{T}=\frac{3}{2} \int_{0}^{T_{m}} R i_{q}^{2} d t+\int_{0}^{T_{m}}\left(A+B \omega+C \omega^{2}\right) \omega d t
$$

Using drive's parameters the energy to cover losses is described as

$$
\begin{aligned}
& W_{T}=\frac{\theta_{r d}^{2}}{\left(T_{m}-T_{\varepsilon}\right)^{2}}\left[\frac{k_{1}}{T_{\varepsilon}}+\frac{3 R_{s} A^{2}}{2 c \Psi^{2}} T_{m}+k_{2}\left(T_{m}-\frac{4}{3} T_{\varepsilon}\right)\right. \\
& +k_{3} \frac{\theta_{r d}^{2}}{\left(T_{m}-T_{\varepsilon}\right)^{2}}\left(T_{m}-\frac{8}{5} T_{\varepsilon}\right) \\
& +k_{4} \frac{\theta_{r d}}{\left(T_{m}-T_{\varepsilon}\right)}\left(T_{m}-\frac{4}{3} T_{\varepsilon}\right) \\
& \left.+k_{5} \frac{\theta_{r d}}{\left(T_{m}-T_{\varepsilon}\right)}\left(T_{m}-\frac{3}{2} T_{\varepsilon}\right)+A \theta_{r d}\right],
\end{aligned}
$$

in which $k_{1}=3 R\left(J_{r}^{2} / c \Psi^{2}\right), k_{2}=(3 / 2) R\left(B^{2} / c \Psi^{2}\right)+B, k_{3}=$ $(3 / 2) R\left(C^{2} / c \Psi^{2}\right), k_{4}=3 R\left(A C / c \Psi^{2}\right), k_{5}=3 R\left(B C / c \Psi^{2}\right)+C$, and $k_{s}=k_{2}+k_{4}$. 


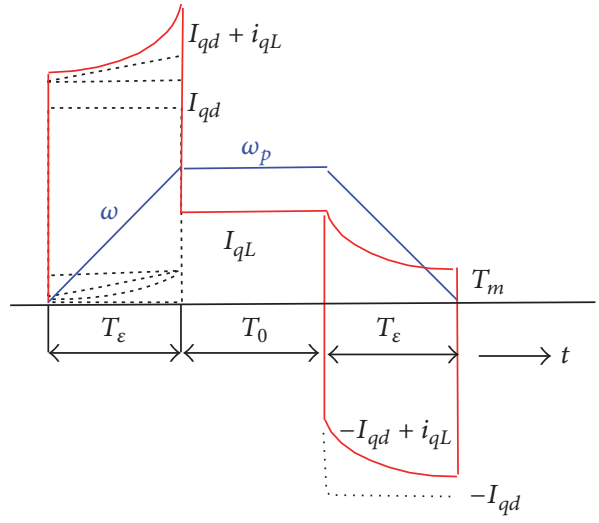

FIGURE 2: Selected speed profile, $\omega_{r}(t)$, and corresponding motor currents.

Optimized acceleration time, $T_{\varepsilon}$, for energy demands minimization is determined as the derivative, $d W_{T} / d T_{\varepsilon}=0$ :

$$
\begin{aligned}
& \frac{-4}{3} k_{s} T_{\varepsilon}^{5}+\left[\frac{10}{3} k_{s} T_{m}+3 k_{5} \theta_{r d}\right] T_{\varepsilon}^{4} \\
& +\left[3 k_{1}-\frac{2}{3} k_{s} T_{m}^{2}-\frac{9}{2} k_{5} \theta_{r d} T_{m}-\frac{24}{5} k_{3} \theta_{r d}^{2}\right] T_{\varepsilon}^{3} \\
& +\left[-7 k_{1}+\frac{2}{3} k_{s} T_{m}^{3}+\frac{3}{2} k_{5} \theta_{r d} T_{m}^{2}+\frac{12}{5} k_{3} \theta_{r d}^{2} T_{m}\right] T_{\varepsilon}^{2} \\
& +5 k_{1} T_{m}^{2} T_{\varepsilon}-k_{1} T_{m}^{3}=0,
\end{aligned}
$$

where (26) is the fifth-order algebraic equation. To find its zeros and value of near-energy optimal acceleration time, $T_{\varepsilon \text { opt }}$, a Newton formula having quadratic convergence is used:

$$
x_{n+1}=x_{n}-\frac{f\left(x_{n}\right)}{f^{\prime}\left(x_{n}\right)}, \quad n=0,1,2, \ldots
$$

Value of near-energy optimal acceleration time, $T_{\varepsilon \text { opt }}$, determines also optimal acceleration, $\varepsilon_{\text {popt }}$, as

$$
\varepsilon_{p \mathrm{opt}}=\frac{\theta_{r d}}{T_{\varepsilon \mathrm{opt}}\left(T_{m}-T_{\varepsilon \mathrm{opt}}\right)} .
$$

During energy consumption analysis it was found that for any speed dependent torque components (linear or quadratic) to decrease energy expenditures means to keep drive's cruising speed to the minimum practicable values. On the other hand, any combination of these two frictions with constant Coulomb friction brings the only one unique solution for maneuver time, $T_{m}$.

To determine also optimal maneuver time, $T_{m}$, for energy expenditures minimization the total energy (25) was expressed in terms of maneuver time as

$$
\begin{aligned}
W_{T}= & k_{1} \frac{\theta_{r d}^{2}}{\kappa(1-\kappa)^{2} T_{m}^{3}}+k_{3} T_{m} \\
& +k_{2} \frac{\theta_{r d}^{2}}{(1-\kappa)^{2} T_{m}}\left(1-\frac{4}{3} \kappa\right)
\end{aligned}
$$

$$
\begin{aligned}
& +k_{4} \frac{\theta_{r d}^{4}}{(1-\kappa)^{4} T_{m}^{3}}\left(1-\frac{8}{5} \kappa\right) \\
& +k_{5} \frac{\theta_{r d}^{3}}{(1-\kappa)^{3} T_{m}^{2}}\left(1-\frac{3}{2} \kappa\right),
\end{aligned}
$$

where coefficient $\kappa$ is defined as a ratio between acceleration and maneuver time, $\kappa=T_{\varepsilon} / T_{m}$. Optimized maneuver time, $T_{m}$, is found again exploiting relation $d W_{T} / d T_{m}=0$ as

$$
\begin{gathered}
\frac{k_{3}(1-\chi)^{2}}{\theta_{r d}^{2}} T_{m}^{4}-k_{2}\left(1-\frac{4}{3} \kappa\right) T_{m}^{2} \\
-\frac{2 k_{5} \theta_{r d}}{(1-\kappa)}\left(1-\frac{3}{2} \kappa\right) T_{m}-\frac{3 k_{1}}{\kappa} \\
-\frac{3 k_{4} \theta_{r d}^{2}}{(1-\kappa)^{2}}\left(1-\frac{8}{5} \kappa\right)=0 .
\end{gathered}
$$

Newton formula (27) with quadratic convergence is again used to find zeros of (30) as energy near-optimal maneuver time. Easy zeros finding is possible due to the fact that if only constant torque is assumed then (26) is reduced into second order only and has analytical solution for acceleration time as $T_{\varepsilon}=T_{m} / 3$. If viscous and quadratic components are added as torque components, then acceleration time becomes shorter. The correct solution of acceleration time is then from interval $\left\langle\begin{array}{ll}0 & T_{m} / 3\end{array}\right\rangle$. Therefore, if this interval is investigated from both sides ( 0 and $\left.T_{m} / 3\right)$ and the same solution is found then it is the only correct solution. If more roots than one are found there simple drawing of the function $T_{\varepsilon}=F(t)$ for $t \in\left\langle 0 T_{m} / 3\right\rangle$ shows the roots. In this case it is necessary to compute energy consumption for every root separately and to choose the root with lowest consumption.

Due to dependence of motor iron losses on rotor speed these losses can be included into presented losses model. Hysteresis losses are proportional to rotor speed and therefore can be included by corresponding enlargement of friction coefficient, $B$, and similarly iron losses due to eddy currents are proportional to the square of rotor speed and therefore can be included as corresponding enlargement of friction coefficient, $C$.

Mutual coupling between optimal maneuver time, $T_{m}$, and acceleration time, $T_{\varepsilon}$, requires the evaluation of (26) and (30) repeatedly, usually three times, to find "true" optimal values of maneuver time, $T_{m}$, and acceleration time, $T_{\varepsilon}$. Advantage of energy near-optimal control with trapezoidal speed profile is relatively simple for implementation because, for example, for constant load torque it requires control on constant torque during acceleration and deceleration intervals and control on constant speed during cruising interval.

To enable comparison of effects of individual friction components on energy optimal (left column) and energy nearoptimal control (right column) Figure 3 shows such effect of constant, $A$, viscous, $B$, and quadratic, $C$ friction components separately. Common requirements for both position control strategies were position set-point, $\theta_{r \mathrm{dem}}=10 \mathrm{rad}$, and 

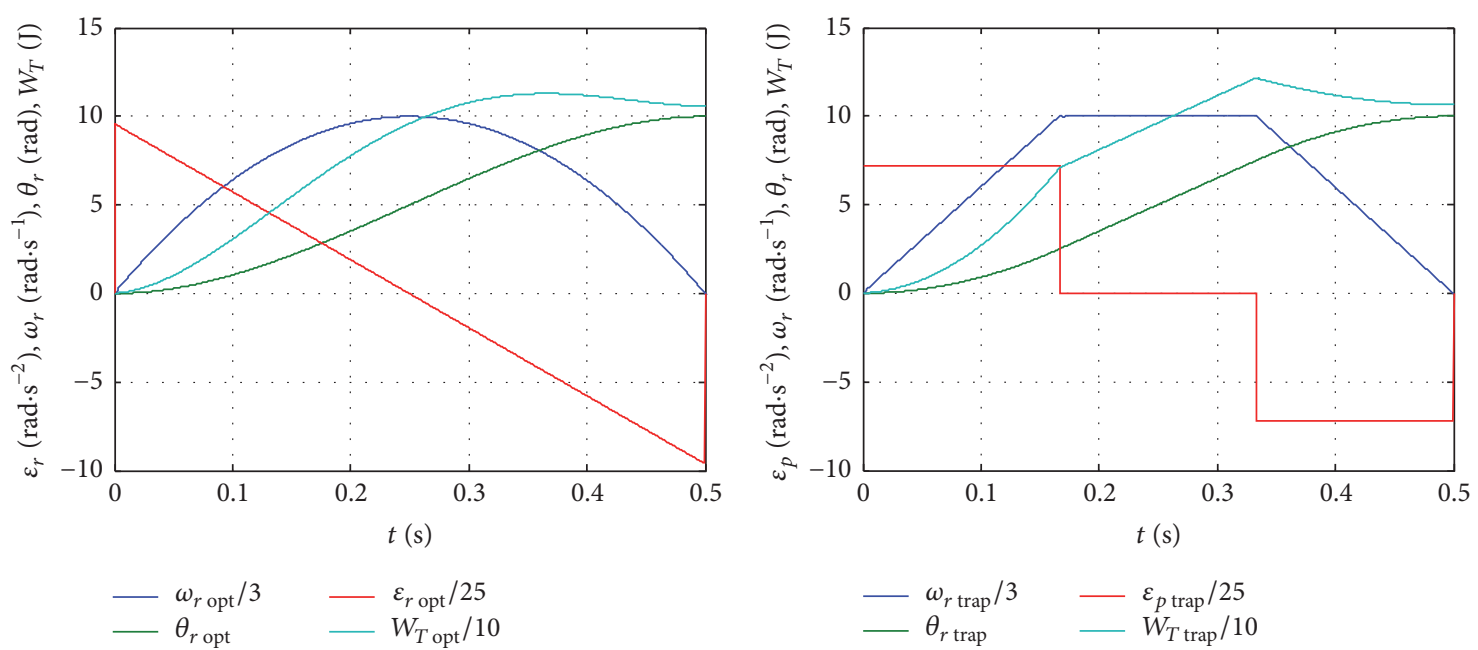

(a) Constant friction component, $A=10 \mathrm{Nm}, B=0$, and $C=0$
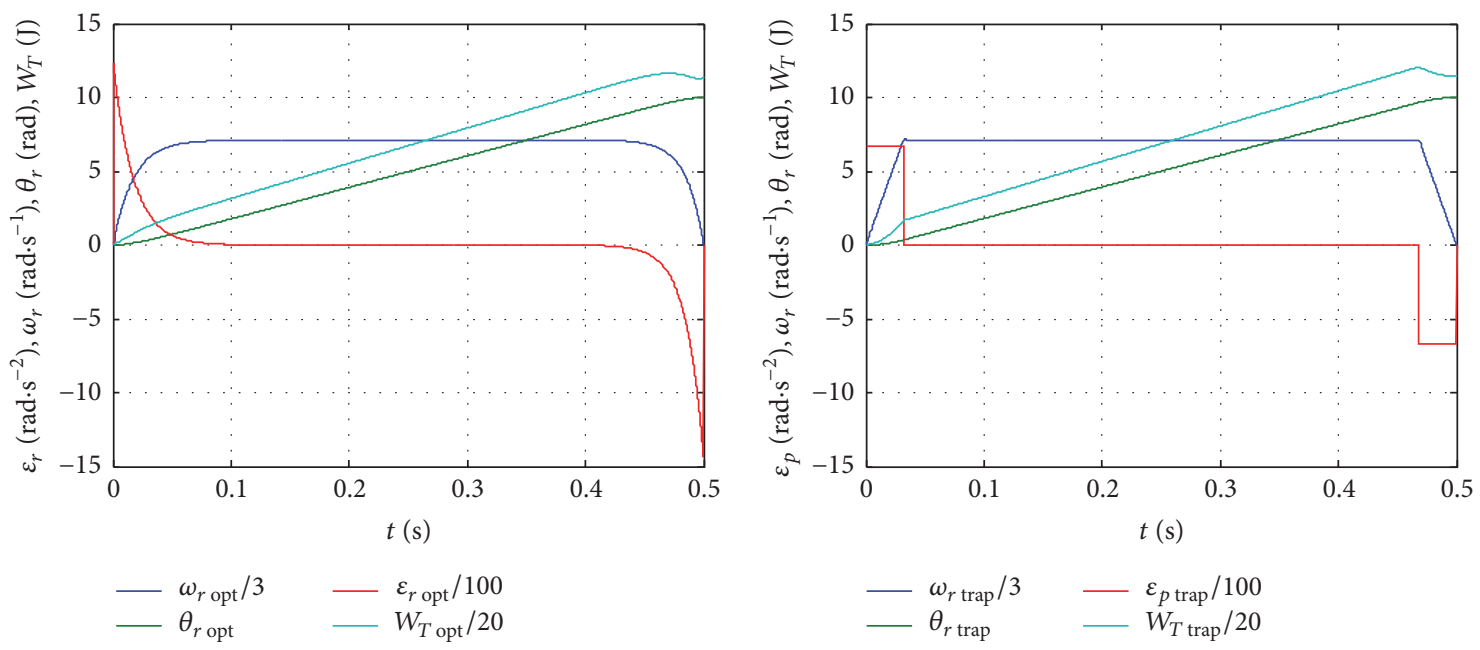

(b) Viscous friction component, $A=0, B=1 \mathrm{Nms}$, and $C=0$
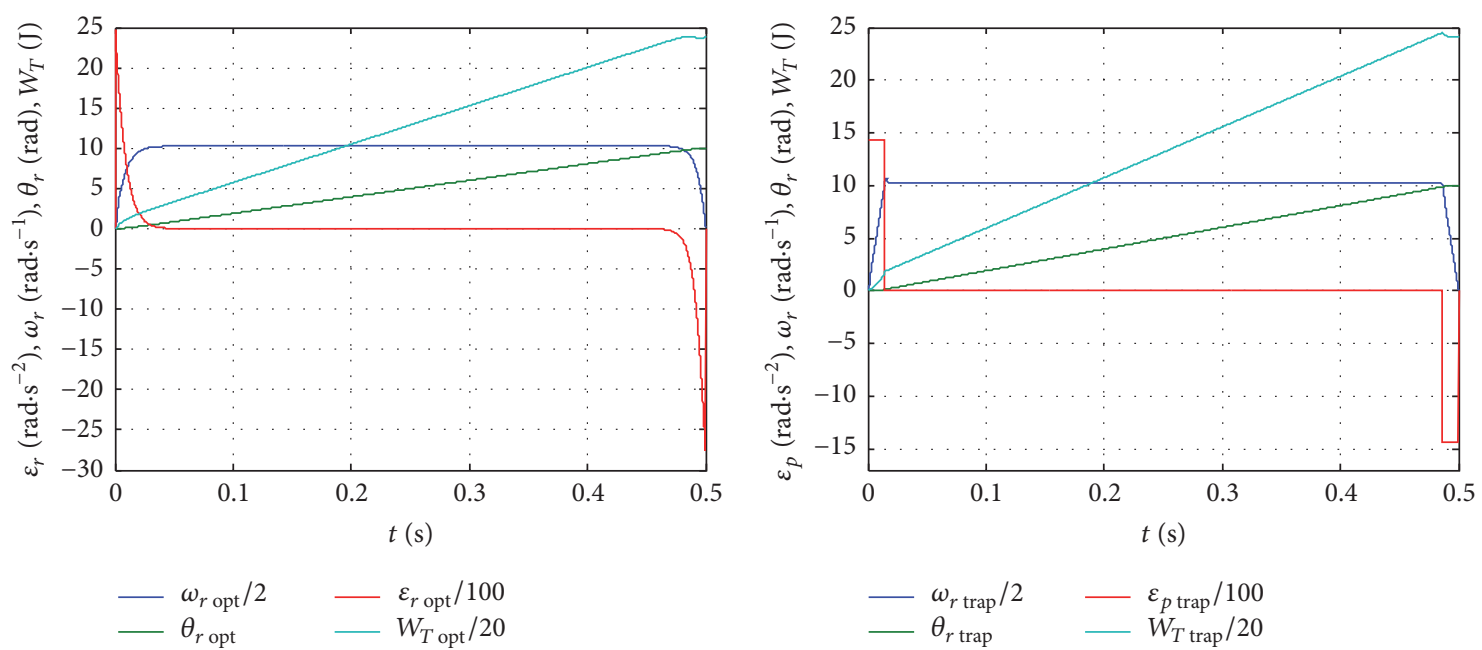

(c) Quadratic friction component, $A=0, B=0$, and $C=0.1 \mathrm{Nms}^{2}$

FIGURE 3: Effect of friction components on profiles of acceleration, speed, position, and energy consumption. 
prescribed maneuver time, $T_{m}=0.5 \mathrm{~s}$. All individual subplots show time functions of rotor acceleration, $\varepsilon_{r}$ (in red), rotor speed, $\omega_{r}$ (in blue), and position, $\theta_{r}$ (in green), together with time profiles of energy consumption, $W_{T}$ (in cyan).

From both subplots (a) showing the effect of constant friction component the same magnitude of cruising speed can be seen. Trapezoidal speed profile for energy nearoptimal control strategy consists of three identical time intervals for acceleration $T_{\varepsilon}=T_{m} / 3$, for constant cruising speed $T_{0}=T_{m} / 3$, and for deceleration $T_{\varepsilon}=T_{m} / 3$, analytically confirmed for description of position system with Coulomb friction only.

From subplots for viscous, subplot (b), and quadratic frictions subplot (c) it is noticeable that for these components times of acceleration and deceleration become shorter and their magnitudes are becoming higher if compared with constant one. On the other hand, time interval of constant cruising speed is getting substantially longer. This feature is exploited further for evaluation of acceleration time of trapezoidal speed profile.

Position time profile for all presented control strategies and types of friction reached required position in prescribed time. Differences in total energy consumption, which are dependent on moment of inertia, for given parameters (moment of drive's inertia was $\mathrm{Jr}=0.0044 \mathrm{kgm}^{2}$ ) are very small. As can be seen from Table 1 the differences between compared control strategies related to values of energy optimal control are from interval $0.5 \%$ up to $1.5 \%$ for constant friction and quadratic friction, respectively.

\section{Design of Position Control System}

For verification of both proposed energy saving control strategies by simulations the control structure consisting of an energy saving profiles generator completed with a zero dynamic lag precompensator and FDC based position control loop capable of precise tracking of prescribed control variables was developed.

3.1. Forced Dynamics Speed Control Loop. Common feature of designed control systems is speed control loop based on FDC while satisfying the vector control conditions [22]. Rotor speed obeys (3), (7) and the differential equation describing the closed loop dynamics has therefore linear firstorder dynamics (31), where $T_{\omega}$ is the prescribed time constant and $\dot{\theta}_{r d}$ is the demanded rotor speed:

$$
\ddot{\theta}_{r}=\frac{1}{T_{\omega}}\left(\dot{\theta}_{r d}-\dot{\theta}_{r}\right) .
$$

The rotor speed derivative equations (3) and (7) for the PMSM and IM may both be written as

$$
\begin{aligned}
\dot{\omega}_{r} & =\frac{1}{J_{r}}\left(c_{m} \boldsymbol{\Psi}^{\mathrm{T}} \mathbf{T}^{\mathrm{T}} \mathbf{I}-\Gamma_{L}\right) \\
& = \begin{cases}\frac{1}{J_{r}}\left[c_{m}\left(\psi_{d} i_{q}-\psi_{q} i_{d}\right)-\Gamma_{L}\right] & \text { for the PMSM } \\
\frac{1}{J_{r}}\left[c_{m}\left(\psi_{D} i_{q}-\psi_{\mathrm{Q}} i_{d}\right)-\Gamma_{L}\right] & \text { for the IM. }\end{cases}
\end{aligned}
$$

For the PMSM, $\Psi_{d}$ and $\Psi_{q}$ are the stator fluxes components and $c_{m}=c=3 p / 2$. For the IM, $\Psi_{D}$ and $\Psi_{Q}$ are the rotor fluxes components and $c_{m}=c_{5}$. Using a switched power electronic control law, the stator current components are made to follow their demands, $i_{d \text { dem }}$ and $i_{q \text { dem }}$, with negligible dynamic lag. Hence, it is assumed that $i_{d}=i_{d \text { dem }}$ and $i_{q}=i_{q \text { dem }}$ which enables the stator current equations to be eliminated from the plant model for the control system design. So for speed control, $i_{d \text { dem }}$ and $i_{q \text { dem }}$ are regarded as control variables. For vector control up to the nominal speed $[23,24], i_{d}=i_{d \text { dem }}=0$ for the PMSM drive and $\Psi_{Q}=0$ for the IM drive, so the terms $\Psi_{q} i_{d}$ and $\Psi_{\mathrm{Q}} i_{d}$ on the RHS of (2) vanish. Equating the RHS of (31) and (32) then yields the same speed control algorithm for both drives:

$$
\begin{aligned}
& i_{d \text { dem }}= \begin{cases}0 & \text { for the PMSM drive } \\
\text { const } \neq 0 & \text { for the IM drive, }\end{cases} \\
& i_{q \text { dem }}=\frac{1}{\psi}\left\{\frac{1}{c_{m}}\left[\frac{J_{r}}{T_{\omega}}\left(\dot{\theta}_{r d}-\dot{\theta}_{r}\right)\right]+\Gamma_{L}\right\},
\end{aligned}
$$

where $\Psi=\Psi_{d}$ for the PMSM drive and $\Psi=\Psi_{D}$ for the IM drive. A load torque observer can be used to estimate the net load torque, $\Gamma_{L}$, on the motor shaft [18].

3.2. Forced Dynamics Position Control Loop. Plant for position control is formed by FDC speed control loop replaced by its ideal transfer function (the first-order lag) and completed with kinematic integrator [25]. Parameters of position control loop are adjusted by application of FDC to this plant using Dodds settling time formula [26]:

$$
\begin{aligned}
\frac{\theta_{r}(s)}{\theta_{r d}^{\prime}(s)} & =\left.\left[\frac{1}{1+s\left(T_{s \theta} / 1.5(1+n)\right)}\right]_{n=2}^{n}\right|_{s \theta} \\
& =\frac{81 / 4 T_{s \theta}^{2}}{s^{2}+\left(9 / T_{s \theta}\right) s+81 / 4 T_{s \theta}^{2}} .
\end{aligned}
$$

If (34) is converted into time domain, it yields the secondorder closed loop differential equation for rotor position:

$$
\ddot{\theta}_{r}=\frac{81}{4 T_{s \theta}^{2}}\left(\theta_{r d}^{\prime}-\theta_{r}\right)-\frac{9}{T_{s \theta}} \dot{\theta}_{r} .
$$

FDC law of rotor angle is obtained by substituting (31) for LHS of (35) and solving this equation for the input variable, which is rotor speed demand, $\dot{\theta}_{r d}$ :

$$
\dot{\theta}_{r d}=\left(1-\frac{9 T_{\omega}}{T_{s \theta}}\right) \dot{\theta}_{r}+\frac{81 T_{\omega}}{4 T_{s \theta}^{2}}\left(\theta_{r d}^{\prime}-\theta_{r}\right) .
$$

3.3. Precompensator and Load Torque Observer Design. Closed loop dynamic of position controller is prescribed by (34); therefore, the precompensator has the inverse transfer function as follows:

$$
F_{\mathrm{PC}}(s)=\frac{\theta_{r d}(s)}{\theta_{r}(s)}=s^{2} \frac{4 T_{s}^{2}}{81}+s \frac{4 T_{s}}{9}+1 .
$$


TABLE 1: Summary of total energy consumption for chosen load torque components.

\begin{tabular}{lcc}
\hline Torque component & Energy optimal control & Energy near-optimal control \\
\hline Constant $A=10 \mathrm{Nm}, B=0, C=0$ & $W_{T \text { opt }}=105.936 \mathrm{~J}$ & $W_{T \text { trap }}=106.433 \mathrm{~J}$ \\
Viscous $A=0, B=1 \mathrm{Nms}, C=0$ & $W_{T \text { opt }}=226.813 \mathrm{~J}$ & $W_{T \text { trap }}=229.5273 \mathrm{~J}$ \\
Quadratic $A=0, B=0, C=0.1 \mathrm{Nms}^{2}$ & $W_{T \text { opt }}=475.604 \mathrm{~J}$ & $W_{T \text { trap }}=482.739 \mathrm{~J}$ \\
\hline
\end{tabular}

Rem.: individual torque coefficients $A, B$, and $C$ were chosen in such a way that they create load torque of $10 \mathrm{Nm}$ for a given cruising speed.

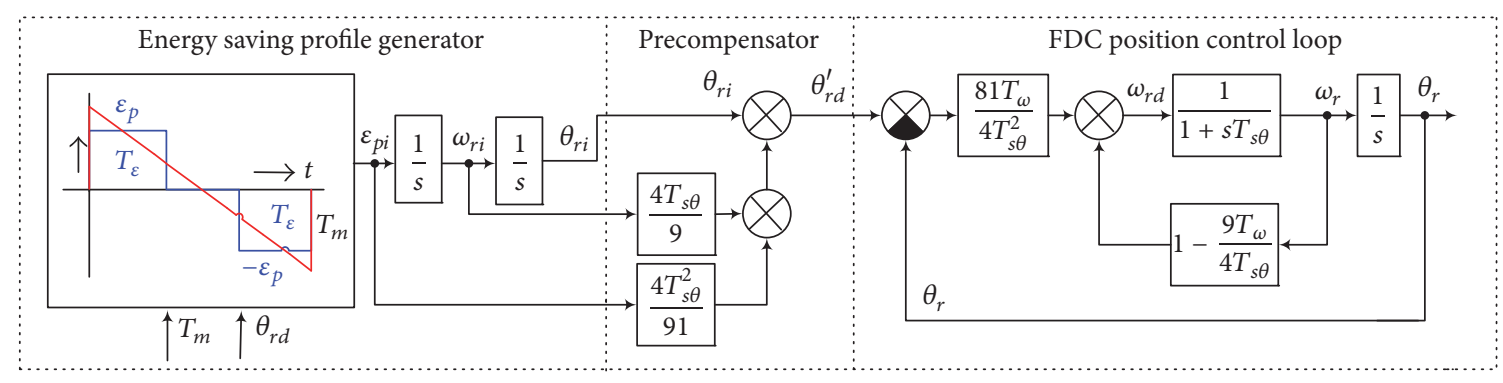

FIGURE 4: Block diagram for energy near-optimal position control strategies.

The overall position control system for energy near-optimal control strategies consisting of energy saving profile generator, precompensator, and FDC position control loop is shown in Figure 4.

To check energy requirements of the derived energy nearoptimal control strategies drive's total energy consumption is evaluated via input power integration as

$$
W_{T}=\frac{3}{2} \int_{0}^{T_{m}}\left(u_{q} i_{q}+u_{d} i_{d}\right) d t .
$$

\section{Stability Analysis of the Closed Loop Systems}

In this section, stability analysis is presented for the feedback control systems for the PMSM and IM drives to ensure that stability is guaranteed with any realistic plant parameter estimation errors that occur in applications.

4.1. Stability Analysis of the PMSM Position Control System. The closed loop systems of both drives are defined by equations (32) and (4) or (8) together with control law (33) with $i_{d \text { dem }}=0$. As the plant has two state variables that could be chosen as $\theta_{r}$ and $\omega_{r}$, and the control law is nondynamic, the closed loop system exhibits second-order dynamics with no internal zero dynamics [26]. Since it is forced by the control law to obey the closed loop differential equation (35), it is a linear, time invariant system with the characteristic equation:

$$
\begin{aligned}
s^{2}+\frac{9}{T_{s \theta}} s+\frac{81}{4 T_{s \theta}^{2}} & =0 \Longrightarrow \\
\left(s+\frac{4.5}{T_{s \theta}}\right)^{2} & =0 .
\end{aligned}
$$

The closed loop poles are therefore $s_{1,2}=-4.5 / T_{s \theta}$ and since they lie in the left half of the $s$-plane, the system is stable.
In practice, however, the plant parameters cannot be known exactly and so analysis will now be carried out to ensure that the system remains stable with plant modelling errors within realistic limits. With $i_{d}=i_{d \text { dem }}=0, i_{q}=i_{q \text { dem }}$, and, from Section 2, $\psi_{d}=L_{d} i_{d}+\psi_{\mathrm{pm}}=\psi_{\mathrm{pm}}$ plant equation (32) combined with (8) becomes

$$
\dot{\omega}_{r}=\frac{1}{J_{r}}\left[c_{m}\left(\psi_{D} i_{q \mathrm{dem}}\right)-\Gamma_{L}\right]
$$

and this may be simplified to

$$
\ddot{\theta}_{r}=P_{1} i_{q \text { dem }}-P_{3} \Gamma_{L},
$$

where $P_{1}=c_{m} \psi_{\mathrm{PM}} / J_{r}$ and $P_{3}=1 / J_{r}$ are constant plant parameters.

FDC control law (33) may be then written as

$$
i_{q \mathrm{dem}}=\frac{1}{\widetilde{P}_{1}}\left[\frac{1}{T_{\omega}}\left(\dot{\theta}_{r d}-\dot{\theta}_{r}\right)+\widetilde{P}_{3} \Gamma_{L}\right],
$$

where $\widetilde{P}_{1}$ and $\widetilde{P}_{3}$ are, respectively, estimates of $P_{1}$ and $P_{3}$. The closed loop differential equation of the position control loop with the plant modelling errors is then obtained by substituting for $i_{q \mathrm{dem}}$ in (41) using (42) and then for $\dot{\theta}_{r d}$ using (36). Thus,

$$
\begin{aligned}
\ddot{\theta}_{r}= & \frac{P_{1}}{\widetilde{P}_{1}} \frac{1}{T_{\omega}}\left(\dot{\theta}_{r \mathrm{dem}}-\dot{\theta}_{r}\right)+\left(\frac{P_{1}}{\widetilde{P}_{1}} \widetilde{P}_{3}-P_{3}\right) \Gamma_{L} \\
= & \frac{P_{1}}{\widetilde{P}_{1}} \frac{1}{T_{\omega}}\left[\left(1-\frac{9 T_{\omega}}{T_{s \theta}}\right) \dot{\theta}_{r}+\frac{81 T_{\omega}}{4 T_{s \theta}^{2}}\left(\theta_{r d}^{\prime}-\theta_{r}\right)-\dot{\theta}_{r}\right] \\
& +\left(\frac{P_{1}}{\widetilde{P}_{1}} \widetilde{P}_{3}-P_{3}\right) \Gamma_{L} \Longrightarrow
\end{aligned}
$$




$$
\begin{aligned}
\ddot{\theta}_{r} & +\frac{P_{1}}{\widetilde{P}_{1}} \frac{9}{T_{s \theta}} \dot{\theta}_{r}+\frac{P_{1}}{\widetilde{P}_{1}} \frac{81}{4 T_{s \theta}^{2}} \theta_{r} \\
& =\frac{P_{1}}{\widetilde{P}_{1}} \frac{81}{4 T_{s \theta}^{2}} \theta_{r d}^{\prime}+\left(\frac{P_{1}}{\widetilde{P}_{1}} \widetilde{P}_{3}-P_{3}\right) \Gamma_{L} .
\end{aligned}
$$

The closed loop characteristic equation is therefore

$$
s^{2}+\frac{P_{1}}{\widetilde{P}_{1}} \frac{9}{T_{s \theta}} s+\frac{P_{1}}{\widetilde{P}_{1}} \frac{81}{4 T_{s \theta}^{2}}=0 .
$$

The closed loop poles are expressed as

$$
\begin{aligned}
s_{1,2} & =-\frac{P_{1}}{\widetilde{P}_{1}} \frac{9}{T_{s \theta}} \pm \sqrt{\left(\frac{P_{1}}{\widetilde{P}_{1}}\right)^{2} \frac{81}{4 T_{s \theta}^{2}}-\frac{P_{1}}{\widetilde{P}_{1}} \frac{81}{4 T_{s \theta}^{2}}} \\
& =\frac{P_{1}}{\widetilde{P}_{1}} \frac{9}{T_{s \theta}}\left(1 \mp \sqrt{1-\frac{\widetilde{P}_{1}}{P_{1}}}\right) .
\end{aligned}
$$

Noting that $P_{1}>0$ and therefore assuming $\widetilde{P}_{1}>$ 0 , then if the $\widetilde{P}_{1}>P_{1}$ closed loop poles are complex conjugates with a common negative real part. If $\widetilde{P}_{1}<P_{1}$ then $\sqrt{1-\widetilde{P}_{1} / P_{1}}<1$ and therefore both closed loop poles are negative and real. It may therefore be concluded that, with realistic plant parameter estimation errors, the closed loop system will always be stable. It must be noted, however, that as the magnitude of these errors increases, then the closed loop dynamics will depart further from that specified according to (36).

4.2. Stability Analysis of the IM Position Control System. Here the closed loop system comprises the plant defined by equations (2), (32), and (4) together with simplified control law (33), where $i_{d}=i_{d \text { dem }}=$ const. $\neq 0$ and $i_{q}=i_{q \text { dem }}$ as the control variable. For vector control of IM in $d_{-q} q$ reference frame coupled to the rotor magnetic flux vector rotating at $\omega_{e}$ the rotor magnetic flux components are $\psi_{D}=L_{m} i_{d}=$ $L_{m} i_{d \text { dem }}=$ const and $\psi_{\mathrm{Q}}=0$; therefore, (2) becomes

$$
\begin{aligned}
& \dot{\psi}_{D}=c_{4} i_{d \mathrm{dem}}-c_{3} \psi_{D}, \\
& \dot{\psi}_{\mathrm{Q}}=c_{4} i_{q \mathrm{dem}}-p \omega_{\mathrm{sl}} \psi_{D},
\end{aligned}
$$

where $\omega_{\mathrm{sl}}=\omega_{e}-\omega_{r}$ is the IM slip angular frequency. For steady-state the component equations are

$$
\begin{aligned}
\left(c_{4}-c_{3} L_{m}\right) i_{d \mathrm{dem}} & =0, \\
c_{4} i_{q \mathrm{dem}}-p \omega_{\mathrm{sl}} L_{m} i_{d} & =0 .
\end{aligned}
$$

Equation (32) combined with (4) becomes

$$
\ddot{\theta}_{r}=\frac{1}{J_{r}}\left[c_{m}\left(\psi_{D} i_{q \mathrm{dem}}\right)-\Gamma_{L}\right]
$$

and this may be again simplified to

$$
\ddot{\theta}_{r}=P_{1} i_{q \mathrm{dem}}-P_{3} \Gamma_{L},
$$

where $P_{1}=c_{m} \psi_{D} / J_{r}$, noting that up to nominal speed $\psi_{D}$ is constant and $P_{3}=1 / J_{r}$ are constant plant parameters.

Then control law (33) becomes

$$
i_{q \mathrm{dem}}=\frac{1}{\widetilde{P}_{1}}\left[\frac{1}{T_{\omega}}\left(\dot{\theta}_{r \mathrm{dem}}-\dot{\theta}_{r}\right)+\widetilde{P}_{3} \Gamma_{L}\right],
$$

where $\widetilde{P}_{1}$ and $\widetilde{P}_{3}$ are, respectively, estimates of $P_{1}$ and $P_{3}$.

In this case the IM closed loop differential equation is identical with (44) of PMSM. Thus,

$$
\begin{aligned}
\ddot{\theta}_{r} & +\frac{P_{1}}{\widetilde{P}_{1}} \frac{9}{T_{s \theta}} \dot{\theta}_{r}+\frac{P_{1}}{\widetilde{P}_{1}} \frac{81}{4 T_{s \theta}^{2}} \theta_{r} \\
& =\frac{P_{1}}{\widetilde{P}_{1}} \frac{81}{4 T_{s \theta}^{2}} \theta_{r \text { dem }}^{\prime}+\left(\frac{P_{1}}{\widetilde{P}_{1}} \widetilde{P}_{3}-P_{3}\right) \Gamma_{L} .
\end{aligned}
$$

The closed loop characteristic equation of IM corresponds to (45) with closed loop poles placed at

$$
\begin{aligned}
s_{1,2} & =-\frac{P_{1}}{\widetilde{P}_{1}} \frac{9}{T_{s \theta}} \pm \sqrt{\left(\frac{P_{1}}{\widetilde{P}_{1}}\right)^{2} \frac{81}{4 T_{s \theta}^{2}}-\frac{P_{1}}{\widetilde{P}_{1}} \frac{81}{4 T_{s \theta}^{2}}} \\
& =\frac{P_{1}}{\widetilde{P}_{1}} \frac{9}{T_{s \theta}}\left(1 \mp \sqrt{\left.1-\frac{\widetilde{P}_{1}}{P_{1}}\right) .}\right.
\end{aligned}
$$

Noting again that $P_{1}>0$ and therefore assuming $\widetilde{P}_{1}>0$, then if the $\widetilde{P}_{1}>P_{1}$ closed loop poles are complex conjugates with a common negative real part. If $\widetilde{P}_{1}<P_{1}$ then $\sqrt{1-\widetilde{P}_{1} / P_{1}}<1$ and therefore both closed loop poles are negative and real. Therefore, for IM it may be also concluded that with, realistic plant parameter estimation errors, the closed loop system will always be stable including remark that as the magnitude of these errors increases then the closed loop dynamics will depart further from that specified according to (36).

\section{Energy Near-Optimal Control Strategies Verification}

Described control strategies for energy expenditures minimization were verified by simulations and preliminary experiments. For simulations the position control system of Figure 4 was used. To achieve higher precision of consumed energy calculation in simulations the load torque was taken as known variable to avoid influence of delay in its estimation. Described control strategies were implemented for simulations of industrial drive employing IM of nominal power $10 \mathrm{~kW}$ and for suburban train unit with two PMSM traction motors, total nominal power $840 \mathrm{~kW}$. Parameters of both motors are listed in the Appendix. For better comparison of the results gained the individual subplots show corresponding control state-space variables for energy near-optimal control in "red" and for modified energy nearoptimal control (with trapezoidal speed profile) in "blue." Common requirements for industrial drive with IM control were position set-point, $\theta_{r \text { dem }}=10 \mathrm{rad}$, and prescribed maneuver time, $T_{m}=0.5 \mathrm{~s}$. 
TABLE 2: Energy consumptions of all four investigated position control strategies of industrial $10 \mathrm{~kW}$ drive.

\begin{tabular}{lcccc}
\hline Position control & Energy optimal & Trapezoidal speed profile & Limited stator current & Triangular speed profile \\
\hline Energy consumption [J] & 368.238 & 371.930 & 378,829 & 517,936 \\
Consumption comparison [\%] & 100.00 & 101.00 & 102,88 & 140.65 \\
\hline
\end{tabular}

Evaluation in percentage is related to the consumption of energy optimal control.

For better evaluation of the influence of chosen position control strategy on energy consumption results of four position control strategies are investigated. The first set of simulations of Figure 5 shows chosen related variables and energy consumption for described energy optimal and energy near-optimal control strategies.

The second set of simulations of Figure 6 shows influence of acceleration and deceleration adjustment when for prescribed maneuver time and given parameters this time was chosen twice longer resulting in half magnitude of acceleration if compared with values prescribed for nearenergy optimal trapezoidal speed profile. Finally position control strategy based on triangular speed profile completes this set. Load torque coefficients of $10 \mathrm{~kW}$ industrial drive were adjusted to create nearly nominal load torque at cruising speed as $A=10 \mathrm{Nm} ; B=0.5 \mathrm{Nms} ; C=0.03 \mathrm{Nms}^{2}$.

Profiles of state variables (rotor acceleration, speed and position, and stator current torque component) as a function of time are shown in subplots (a)-(d). Both designed control strategies reach demanded position set-point, $\theta_{r d}$, in prescribed maneuver time, $T_{m}$, as it is shown in subplot (c). Subplot (d) shows optimized torque components of stator current where the change of current torque component of energy near-optimal control with trapezoidal speed profile follows change of load torque as a function of rotor speed. Subplot (e) shows rotor speed as a function of its position and subplot (f) shows profile of energy consumption as a function of time.

As can be seen from subplot (a) of Figure 5 the required magnitude of energy optimal acceleration reaches nearly $2000 \mathrm{rads}^{-2}$ while magnitude of energy near-optimal control is less than half of this value. As it was supposed during this control strategy development this value for trapezoidal speed profile is constant. Subplot (b) shows time profiles of rotor speed as it was computed by both control strategies. As can be seen from this subplot, maximum of rotor speed during cruising interval is nearly identical with peak of rotor speed of energy near-optimal control. The difference appears if both speeds are compared as a function of position in subplot (e).

Energy consumption for complete maneuver shown in subplot (f) reaches $W_{T \text { opt }}=368.238 \mathrm{~J}$ for ideal energy optimal control and $W_{T \text { trap }}=371.930 \mathrm{~J}$ (with precision of $\mathrm{mJ}$ ) for position control with trapezoidal speed profile. The difference in consumption is $\Delta W_{T}=3,692 \mathrm{~J}$ only, which is very small not only for good tracking of the energy optimal speed profile with trapezoidal one but also due to the fact that two energy near-optimal control strategies are compared.

Simulation results of position control based on limited IM stator current (in black) and position control with triangular speed profile (in green) are shown in Figure 6. As can be seen from subplot (a) of this figure the required magnitude of acceleration due to limited IM current was decreased to the value less than $500 \mathrm{rads}^{-2}$ while acceleration magnitude of triangular speed profile is the lowest (approximately 1/10 of acceleration for energy optimal value). For both presented position control systems these acceleration are constant to produce expected time profiles of rotor speed and position shown as subplot (b) and subplot (c). Stator current torque components of subplot (d) are changing according to load torque changes, due to their dependence on of rotor speed.

As shown in subplots (f) total energy consumption for limited stator current control reached $W_{T \text { curr }}=378.829 \mathrm{~J}$ and for triangular speed profile the consumption was $W_{T \operatorname{trn}}=$ 517.936 J. The difference in energy consumption of these two position control strategies is significant $\Delta W_{T}=139,107 \mathrm{~J}$ and stresses influence of the choice of position control strategy on total energy consumption for rest-to-rest maneuver.

Total energy consumption of position rest-to-rest maneuver for $10 \mathrm{~kW}$ industrial drive employing induction motor with moment of inertia related to motor shaft, $J_{r}=0.09 \mathrm{kgm}^{2}$, under all four investigated control strategies is summarized in Table 2 from which it can be seen that selection of improper position control strategy can increase total energy consumption by 40 percent.

For traction applications all further computations are related to the one traction motor (TM) parameters which are listed in Appendix. The relation between traction unit velocity, $V_{V}$, and TM rotational motor speed, $\omega_{m}$, as well as relation between unit traction force, $F_{t}$, and torque of TM, $\Gamma_{\mathrm{TM}}$, is defined as

$$
\begin{gathered}
V_{V}=1.8 \frac{D_{w a} \omega_{m}}{u}, \\
F_{t}=\frac{m \Gamma_{\mathrm{TM}} 2 u}{D_{w a}},
\end{gathered}
$$

where $D_{w a}$ is average diameter of the driving wheel and $u$ is gear ratio between rotor of TM and driving wheel and $m$ is number of TMs of the train unit. Passive traction resistance is function of vehicle speed as

$$
p_{0}=A+B V_{V}+C V_{V}^{2}
$$

and for suburban unit has the form: $p_{0}=3.1+0.00038 V_{V}^{2}$. For simulations the train movement is described by differential equation as

$$
m_{t} \frac{d^{2} l_{t}}{d t^{2}}=F_{t}(t, V)-F_{p}(V)-F_{s}(l)-F_{b}(V)
$$

where $m_{t}$ is total mass of train, $l_{t}$ is distance, $F_{t}$ is traction force, $F_{p}$ is force covering vehicle resistances, $F_{s}$ is force covering slope resistance, and $F_{b}$ is braking force, respectively. 


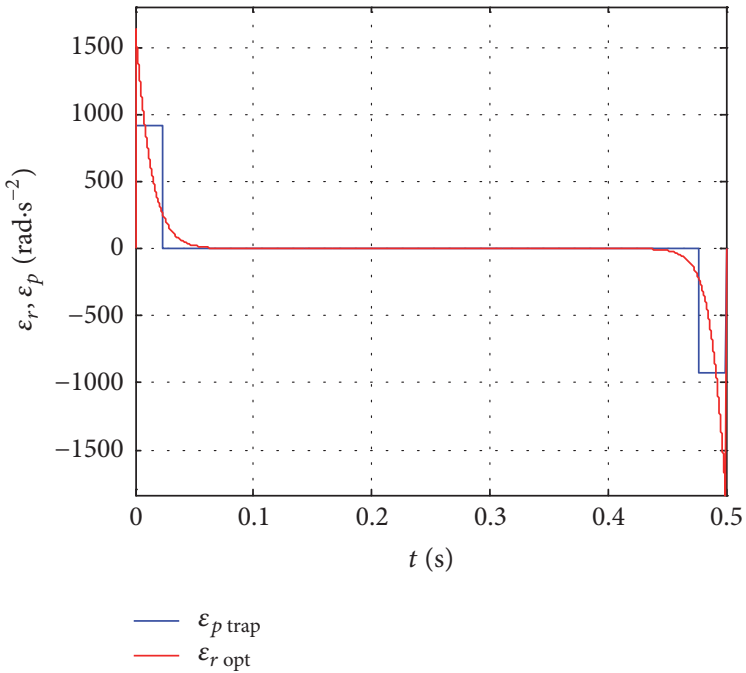

(a) Acceleration

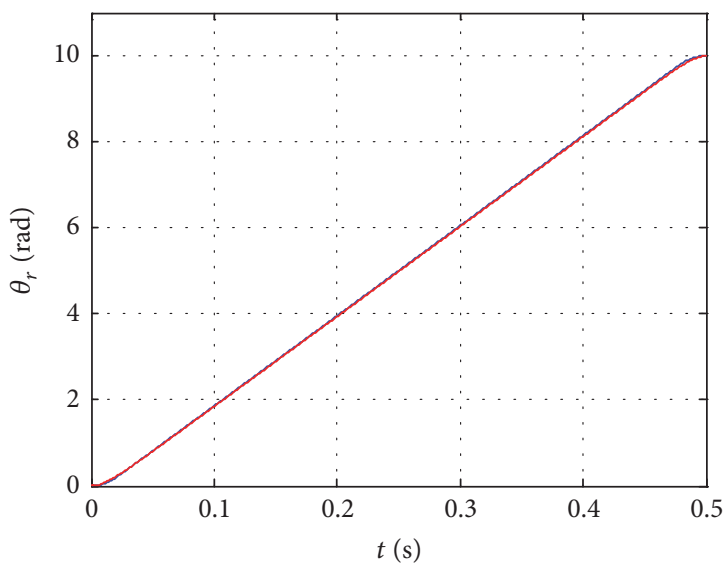

$$
-\theta_{r \text { trap }}
$$

(c) Rotor position

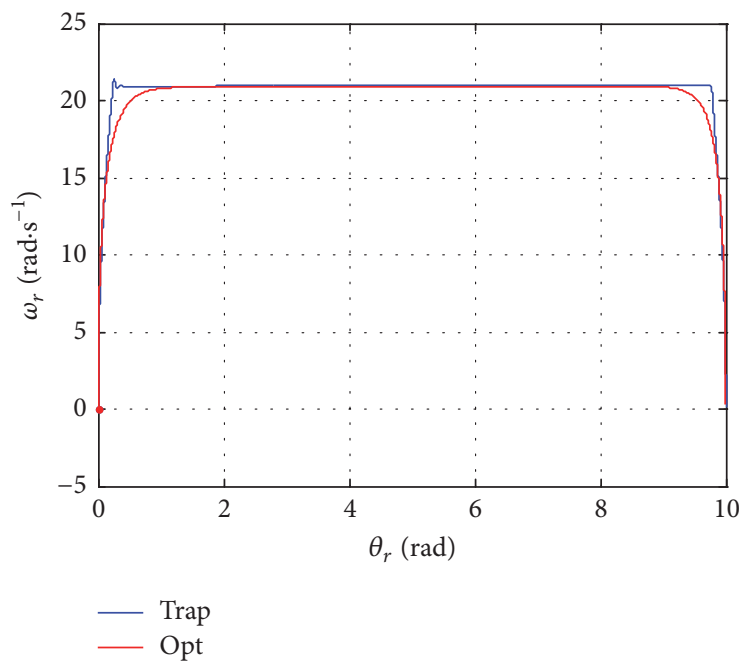

(e) Rotor speed as a function of position

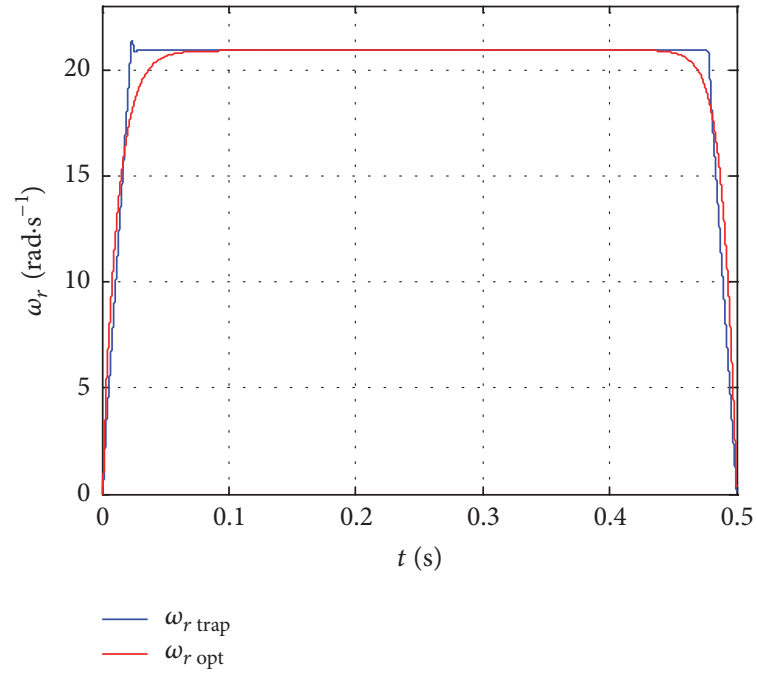

(b) Rotor speed

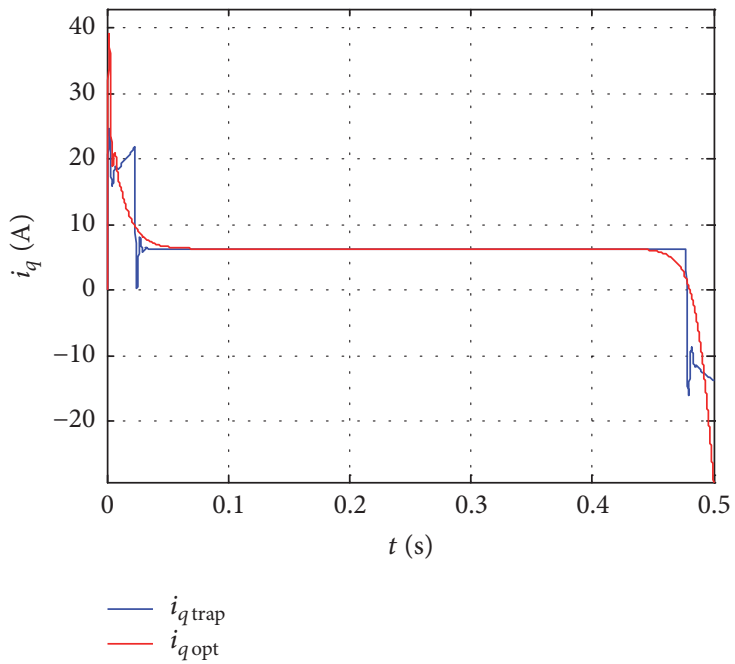

(d) Current torque component

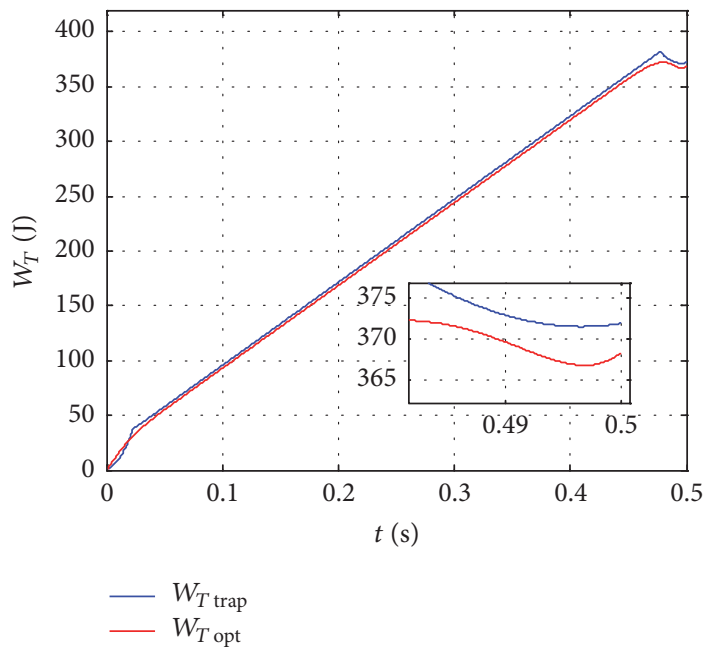

(f) Energy consumption

FIGURE 5: Near-optimal reference acceleration, speed, position, current, and energy consumption of industrial IM $10 \mathrm{~kW}$ drive. 


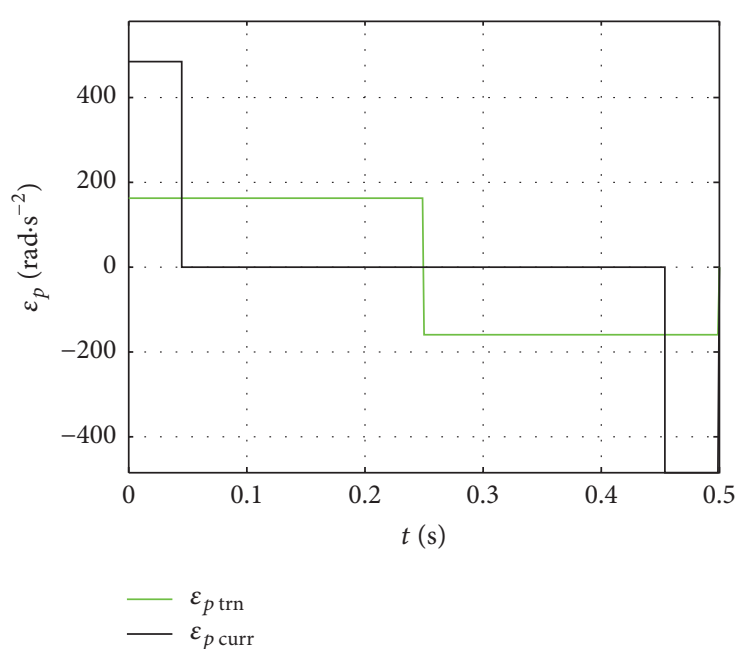

(a) Acceleration

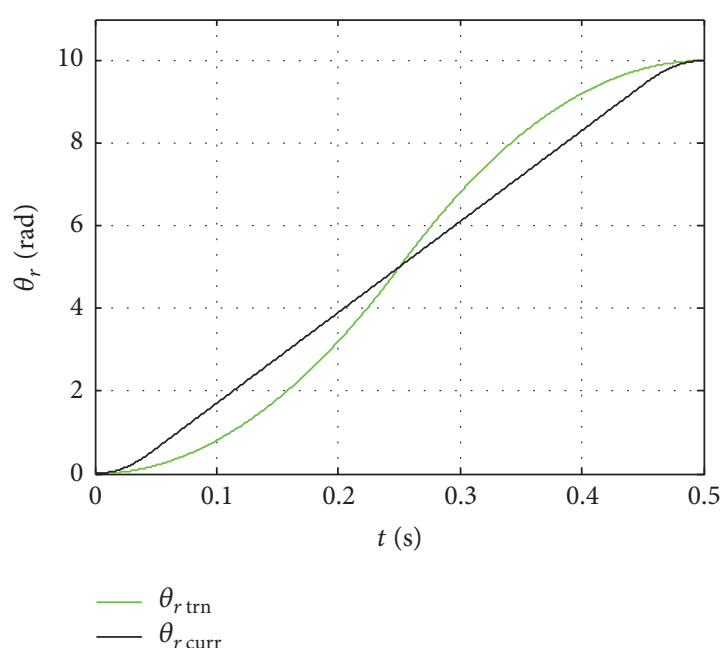

(c) Rotor position

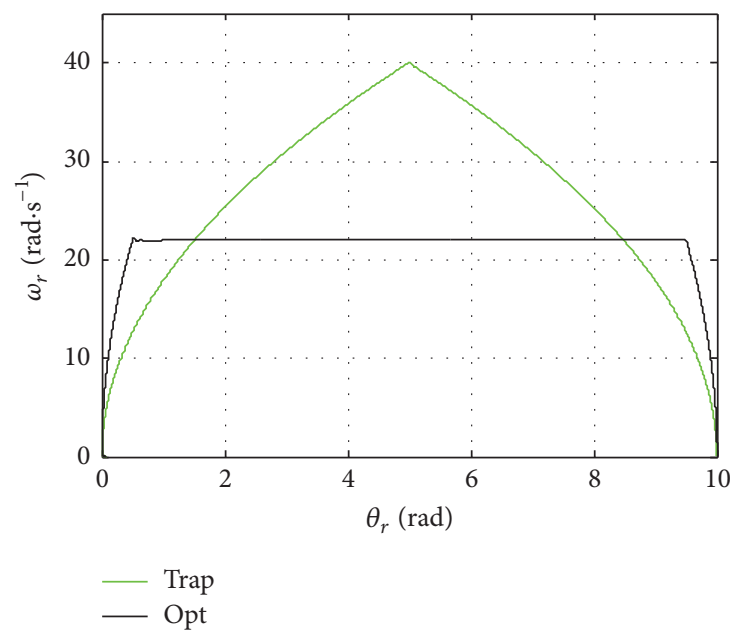

(e) Rotor speed as a function of position

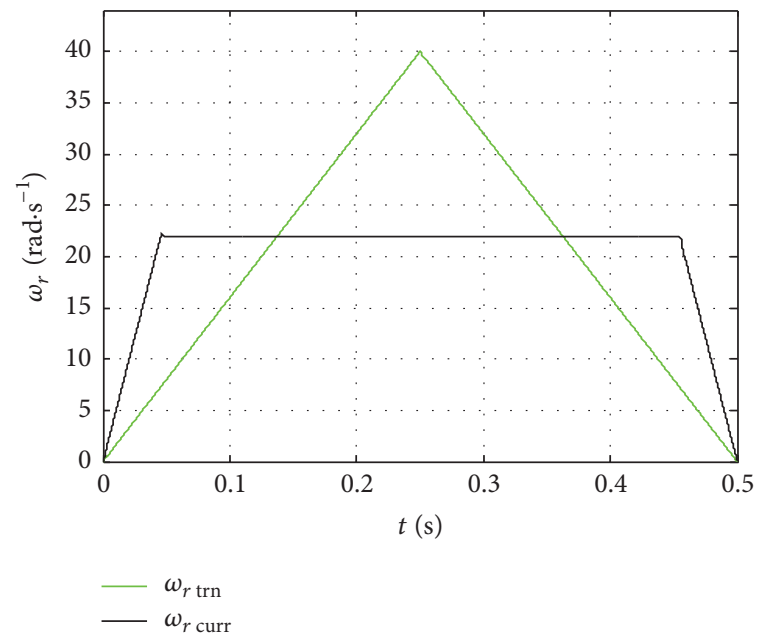

(b) Rotor speed

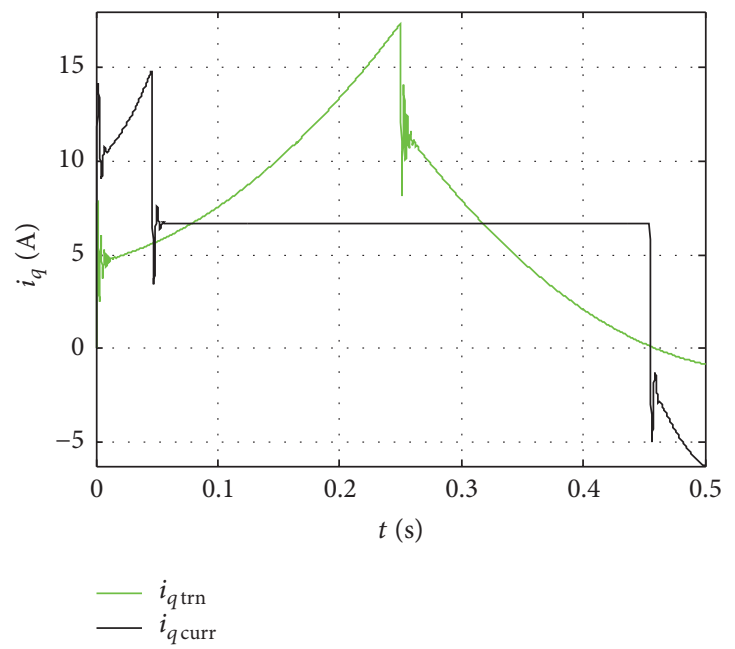

(d) Current torque component

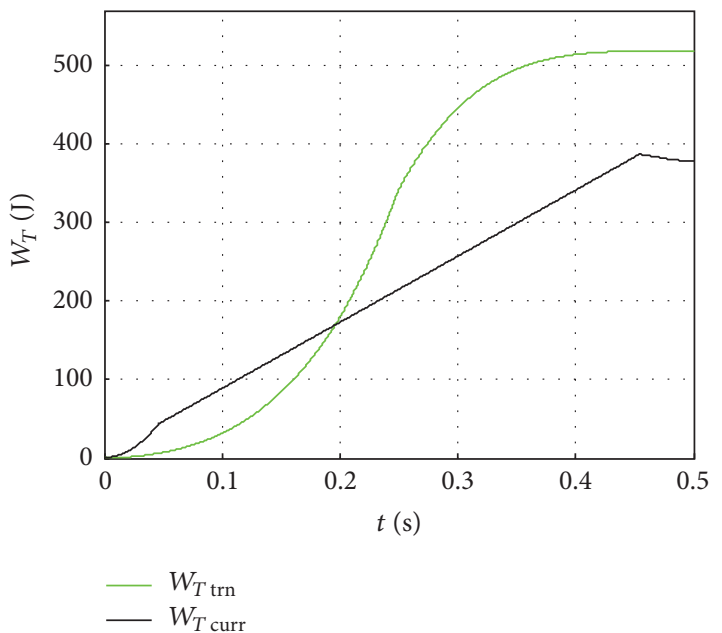

(f) Energy consumption

FIGURE 6: Reference acceleration, speed, and energy consumption of industrial drive for energy consumption evaluation. 
Simulation results for one traction drive are arranged in similar way as it was presented for industrial one. Profiles of state variables (TM acceleration, speed, travelled distance, and stator current torque component) as a function of time are shown in subplots (a)-(d). Subplot (e) shows vehicle speed and subplot (f) shows profiles of energy consumption as a function of time. Load torque coefficients were derived from vehicle traction resistance and leveled route. Results of simulations for traction drive are shown in Figures 7 and 8. Common requirements were demanded travel distance between two stops, $l_{t \text { dem }}=3000 \mathrm{~m}$, and prescribed travel time, $T_{T}=180 \mathrm{~s}$.

The first set of simulations of Figure 7 shows results of applications of described energy optimal and energy nearoptimal control strategies (red and blue). The second set of simulations of Figure 8 shows influence of acceleration and deceleration time adjustment when for prescribed travel time and given traction resistance parameters this time was adjusted to correspond nearly to vehicle adhesion limits. Magnitude of acceleration was increased twice for reduced acceleration time into half if compared with values computed for near-energy optimal trapezoidal speed profile. Finally position control strategy based on triangular speed profile again completes this set.

Figure 7 shows state variables of position control and compares the energy expenditures for energy optimal and energy near-optimal control of train unit. The individual subplots are shown as time functions: prescribed acceleration in subplot (a), TM rotor speed in subplot (b), and travelled distance as subplot (c) followed by TM stator current torque component as subplot (d), vehicle longitudinal velocity as subplot (e), and time function of total energy consumption as subplot (f).

As can be seen from subplot (a) of Figure 7 the required magnitude of energy optimal acceleration reaches nearly $5 \mathrm{~ms}^{-2}$ while magnitude of energy near-optimal control is less than $3.5 \mathrm{~ms}^{-2}$. This acceleration for triangular speed profile is lasting nearly one-third of prescribed travelling time due to prevailing constant load torque component, $A$. As it was supposed during control strategy design this value is for trapezoidal speed profile constant. Subplot (b) shows time profiles of TM rotor speed as it was computed for both control strategies. This subplot confirms again that maximum of TM rotor speed during cruising interval is nearly identical with peak of rotor speed of energy optimal control.

Both designed control strategies reached demanded travel distance, $l_{\mathrm{td}}$, in prescribed time between two stops, $T_{T}=$ $180 \mathrm{~s}$, as it is shown in subplot (c). Subplot (d) shows optimized torque components of PMSM stator current for both control strategies, which corresponds to required acceleration and traction resistances. To keep constant acceleration (deceleration) of the drive the current torque components during these intervals follow changes of load torque. Corresponding vehicle speed profiles are shown in subplot (e). Due to quadratic component, $C$, in traction force, the rotor speed profile for energy optimal control is slightly asymmetrical if compared with assumed symmetrical trapezoidal speed profile.
Total energy consumption between two stops as a function of time is shown in subplot (f). It reached $W_{T \text { opt }}=$ 767.56 Wh for tracking of the ideal energy optimal control and $W_{T \text { trap }}=790,54 \mathrm{Wh}$ for position control with trapezoidal speed profile. The difference in consumption is $\Delta W_{T}=$ $22,98 \mathrm{Wh}$ only, which is only $3 \%$ higher if compared with energy optimal control also due to the fact that two energy near-optimal control strategies are compared.

Simulation results of position control based on increased PMSM stator current (in black) and position control with triangular speed profile (in green) are shown in Figure 8.

As can be seen from subplot (a) of this figure the required magnitude of acceleration due to SMPM stator current on adhesion limit was increased more than four times while acceleration magnitude of triangular speed profile is again the lowest. For both position control systems this acceleration is constant to produce expected time profiles of rotor speed. Corresponding speed profiles as a function of time are shown in subplot (b). Time function of travelled distance is shown as subplot (c). Stator current torque components of subplot (d) are changing only slightly due to small changes of load torque as a function of vehicle speed.

Total energy consumption is shown in subplots (f). This consumption for adhesion stator current reached peak value, $W_{\text {T curr }}=1112.14 \mathrm{Wh}$, while consumption of control strategy with triangular speed profile was $W_{T \operatorname{trn}}=829.07 \mathrm{Wh}$. The difference in energy consumption of these two position control strategies is significant $\Delta W_{T}=283.07 \mathrm{Wh}$, putting the stress on importance of the choice of position control strategy for journey between two stops.

As it is clear from the details of subplots (f) of Figures 7 and 8 the lowest energy requirement is energy optimal control followed by modified energy near-optimal control. On the other hand, the control strategy exploiting highest acceleration and deceleration shows highest energy demands.

Total energy consumption between two stops for one TM $420 \mathrm{~kW}$ with moment of inertia related to motor shaft, $J_{r}=470 \mathrm{kgm}^{2}$, of train unit employing PMSMs under all four investigated control strategies is summarized in Table 3. As can be seen the selection of improper position control strategy, in this case acceleration adjusted to adhesion limit, can increase total energy consumption nearly by 45 percent.

Possibility of optimizing maneuver time, $T_{m}$, as described by (30) is shown in Figure 9 for investigated industrial and traction drive. In these $3 \mathrm{D}$ plots the total energy consumption is shown as a function of acceleration time, $T_{\varepsilon}$, and maneuver time, $T_{m}$. This optimization usually results in much longer maneuver time and therefore it can be exploited only for sufficient time between two position maneuvers [27].

Preliminary experimental verification of proposed energy saving control strategies was carried out using laboratory bench consisting of $1.5 \mathrm{~kW}$ IM (parameters listed in Appendix) loaded with synchronous generator supplying constant resistive load. Position control strategies based on derived energy optimal, energy near-optimal (trapezoidal speed profile), and triangular speed profile were implemented to a vector controlled drive with IM. Tracking performances of this drive to follow state variables generated by energy saving profile generator were improved by exploitation of precompensator. 

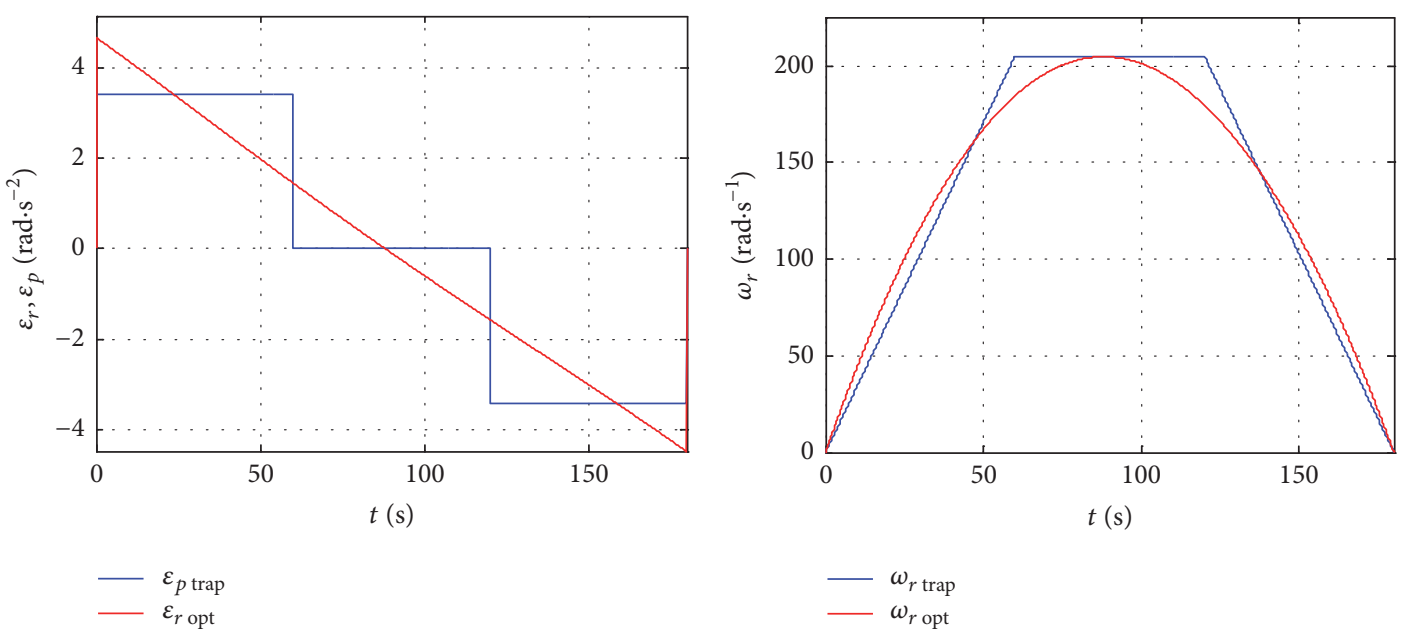

(a) Acceleration
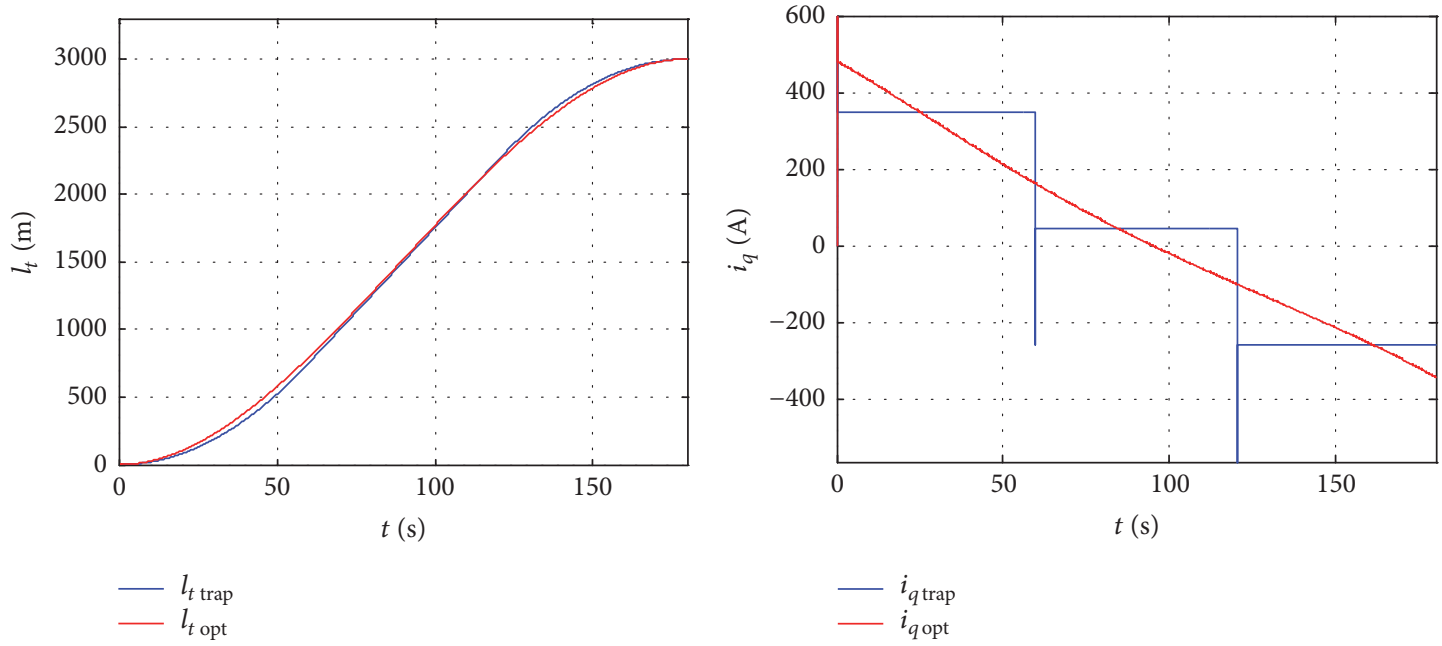

(c) Travel distance

(d) Current torque component
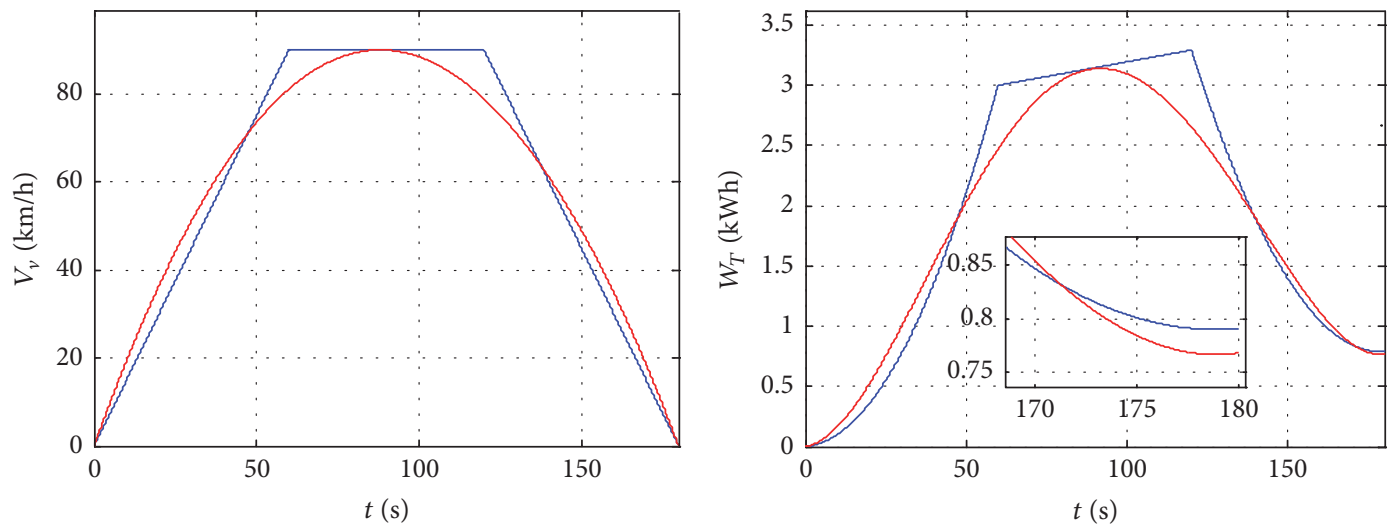

$-V_{v \text { trap }}$

$-W_{T \text { trap }}$

(e) Vehicle velocity

(f) Energy consumption

FIgURE 7: Near-optimal reference acceleration, speed, energy consumption, and travel distance for traction drive. 

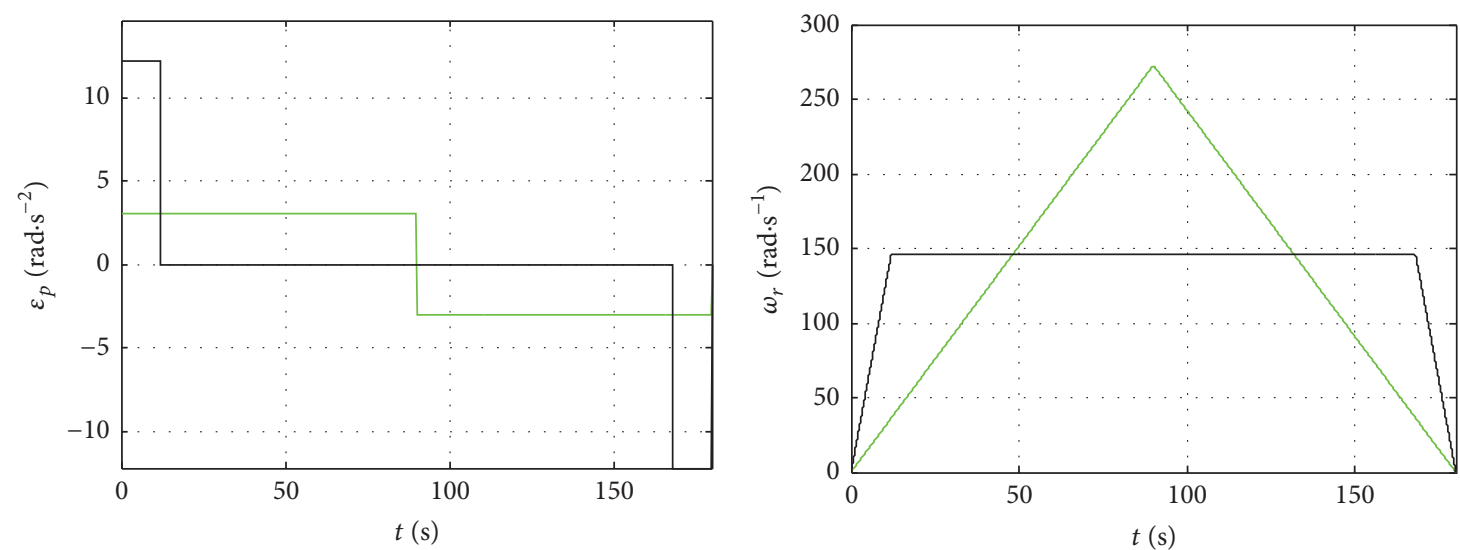

$-\varepsilon_{p \text { trn }}$

$-\omega_{r \operatorname{trn}}$

$-\omega_{r \text { curr }}$

(a) Acceleration
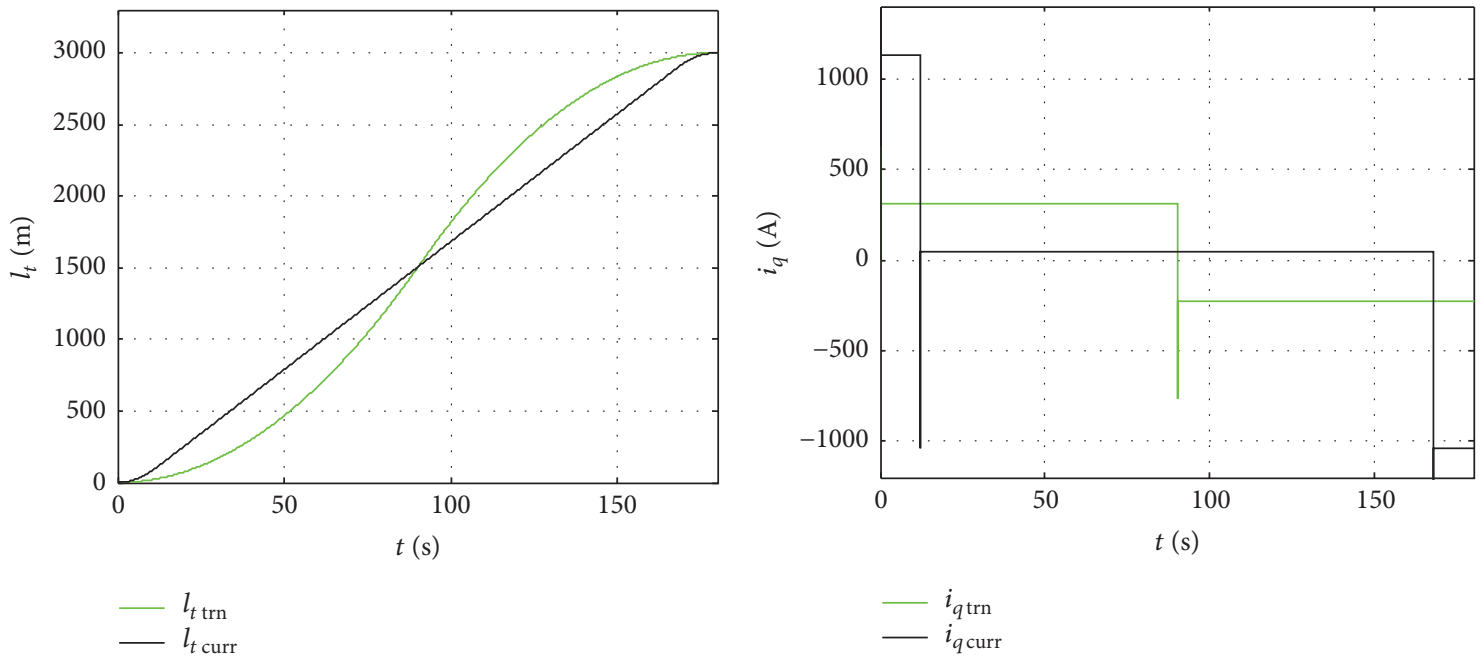

(c) Travel distance

(d) Current torque component
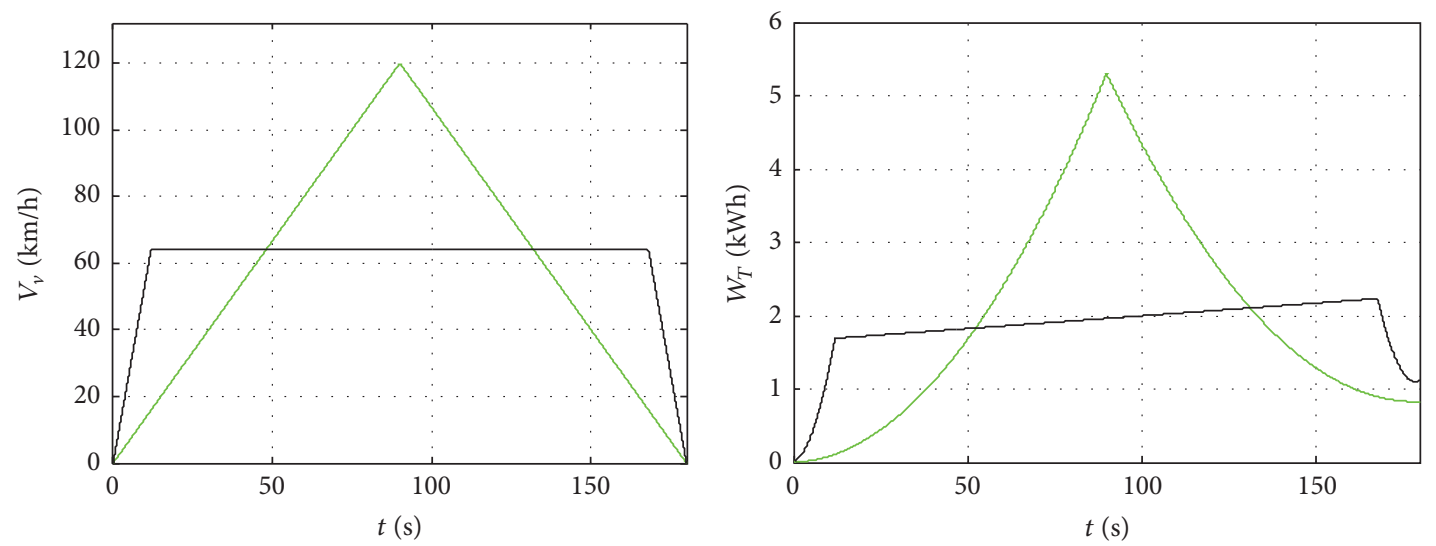

$$
\begin{aligned}
& -V_{v \text { trn }} \\
& -V_{v \text { curr }}
\end{aligned}
$$$$
\begin{aligned}
& -W_{T \operatorname{trn}} \\
& -W_{T \text { curr }}
\end{aligned}
$$

(e) Vehicle velocity

(f) Energy consumption

FIGURE 8: Reference rotor acceleration, speed, travel distance, stator current, vehicle speed, and total energy consumption of traction drive. 


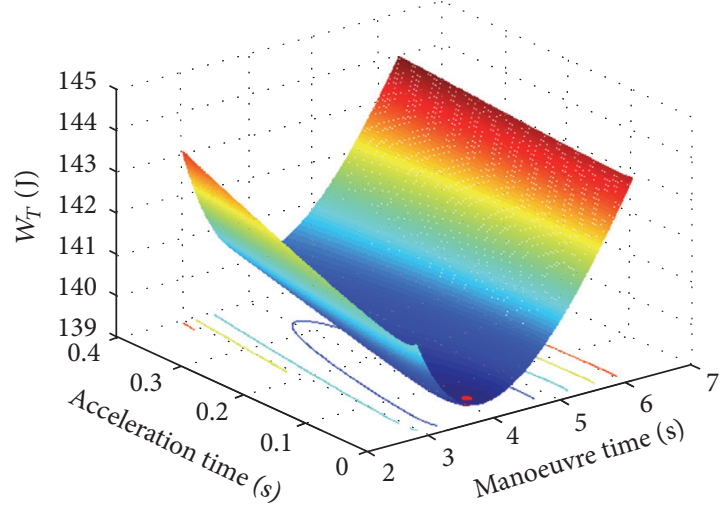

(a) Industrial drive with IM $10 \mathrm{~kW}$

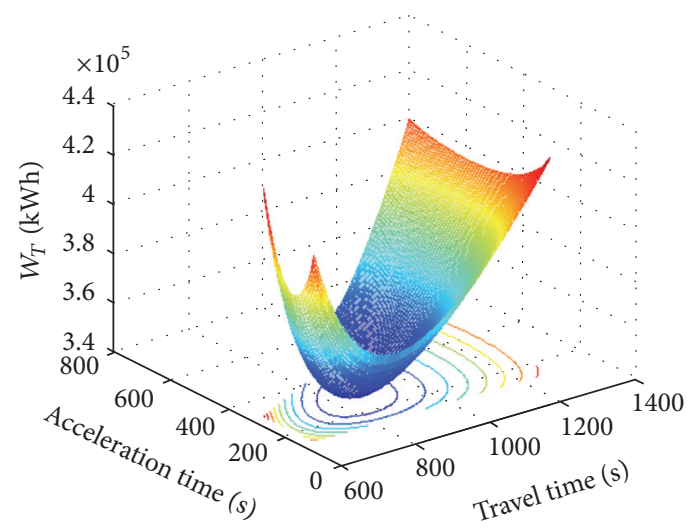

(b) Traction drive with PMSM $420 \mathrm{~kW}$

FIGURE 9: Energy consumption as a function of acceleration time, $T_{\varepsilon}$, and maneuver time, $T_{m}$, and travel time, $T_{T}$, respectively.

TABLE 3: Energy consumption of all four investigated position control strategies for $420 \mathrm{~kW}$ traction drive.

\begin{tabular}{lcccc}
\hline Position control & Energy optimal & Trapezoidal speed profile & Stator current as adhesion limit & Triangular speed profile \\
\hline Consumption of energy [Wh] & 767.56 & 790.54 & 1112.14 & 829.07 \\
Difference in consumption in [\%] & 100.00 & 102.99 & 144.89 & 108.01 \\
\hline
\end{tabular}

Evaluation in percentage is related to the consumption of energy optimal control.

Identification of load on the shaft of IM resulted in constant torque component, $A=0.166 \mathrm{Nm}$, and component proportional to the rotor speed, $B=0.0531 \mathrm{Nms}$ while quadratic component $C=0$. Measured results are shown in Figure 10 .

The individual subplots show (a) time functions of rotor speed, $\omega_{r}(t)$, subplot (b) position profiles, $\theta_{r}(t)$, and subplot (c) time functions of corresponding current torque components $i_{q}(t)$. Total energy consumption, $W_{T}(t)$, for investigated control strategies is shown in subplot (d).

Evaluation of total energy consumption resulted in $W_{T \text { opt }}$ $=14.17 \mathrm{~J}$ for tracking speed profile, which corresponds to energy optimal control strategy. Total energy consumption $W_{T \text { trap }}=14.32 \mathrm{~J}$ was recorded for trapezoidal speed profile of energy near-optimal control and finally $W_{T \operatorname{trn}}=16.91 \mathrm{~J}$ energy consumption corresponds to position control strategy exploiting triangular speed profile. Experimental results show that the lowest energy consumption was achieved by control strategy tracking speed profile of optimal control. Total energy consumption of trapezoidal speed profile is only $1.06 \%$ higher and triangular speed profile has highest energy consumption, which is $19,34 \%$ higher if compared with energy consumption of speed profile of energy optimal position control.

Preliminary experimental results are in good agreement with simulations presented and small differences can be explained by dependence of energy savings on moment of the drive inertia. The differences of 3-5\% between preliminary experiments and subsequently repeated simulations for given parameters were caused by unmodelled friction components and current measurement when measured noise in stator current contributed to the higher value of integrated energy consumption.

\section{Conclusions and Recommendations}

Design and verification of the two energy near-optimal control strategies based on Euler-Lagrange minimization and modified energy near-optimal strategy with trapezoidal speed profile were presented. For comparison of energy savings achieved these two strategies are completed with control strategy based on modified trapezoidal speed profile and position control strategy based on triangular speed profile. All these strategies were applied to rotational industrial drive employing $10 \mathrm{~kW}$ induction motor and to traction unit with $420 \mathrm{~kW}$ PMSM. Control system able to track closely prescribed state variables of energy optimal and energy nearoptimal reference input generator was used in simulations to evaluate the energy expenditures of investigated control strategies. Chosen position control strategies were verified also by preliminary experiments, which confirmed good agreement with presented theory.

The main contribution of this research is a new position control strategy for permanent magnet synchronous motor drives and induction motor drives achieving minimization of frictional and electrical energy losses for specified maneuver times. The novelty lies in avoiding direct implementation of complex nonlinear optimal feedback control laws, in which plant modelling uncertainties could cause unacceptable suboptimal performance or even instability, by instead implementing vector controlled drives with forced dynamic control laws yielding known linear closed loop dynamics, together with a precompensator eliminating dynamic lag, enabling a precomputed easily generated near-optimal reference input function to be accurately followed, thereby forcing the system to follow a near-optimal state trajectory. 


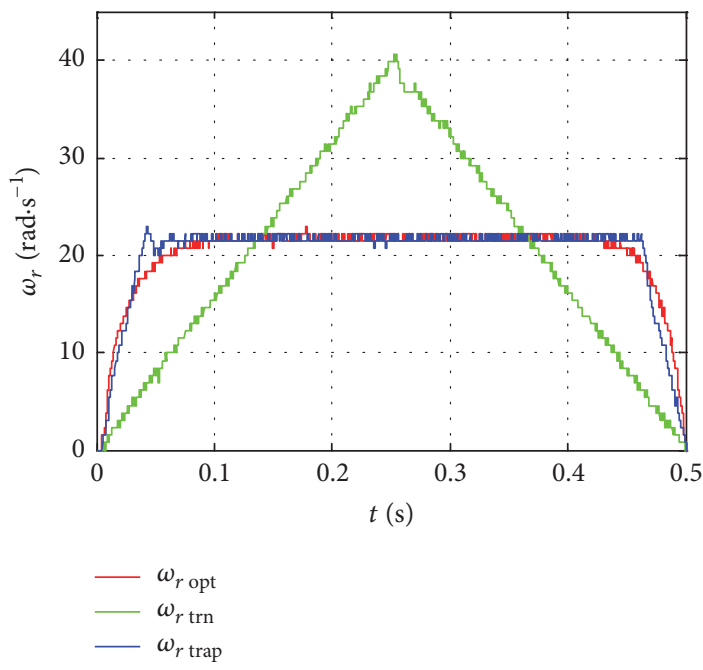

(a) Speed profiles

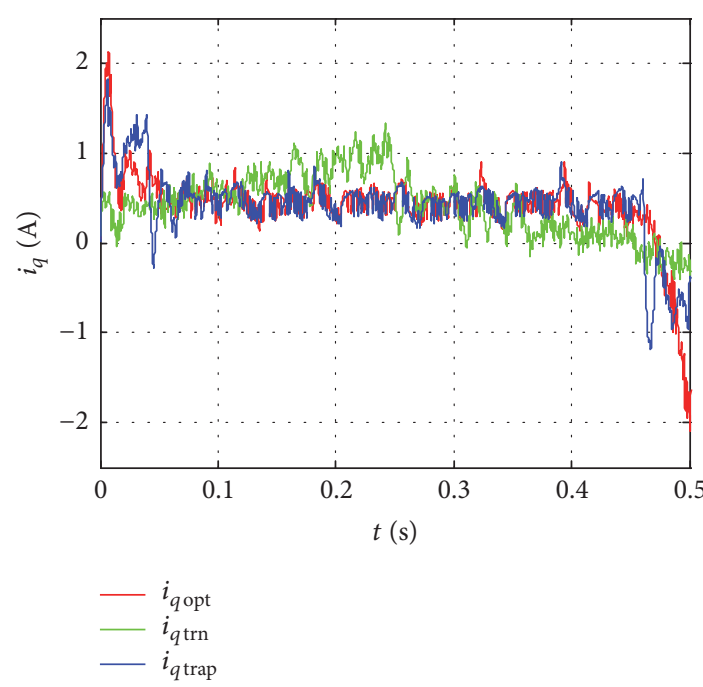

(c) Current torque component

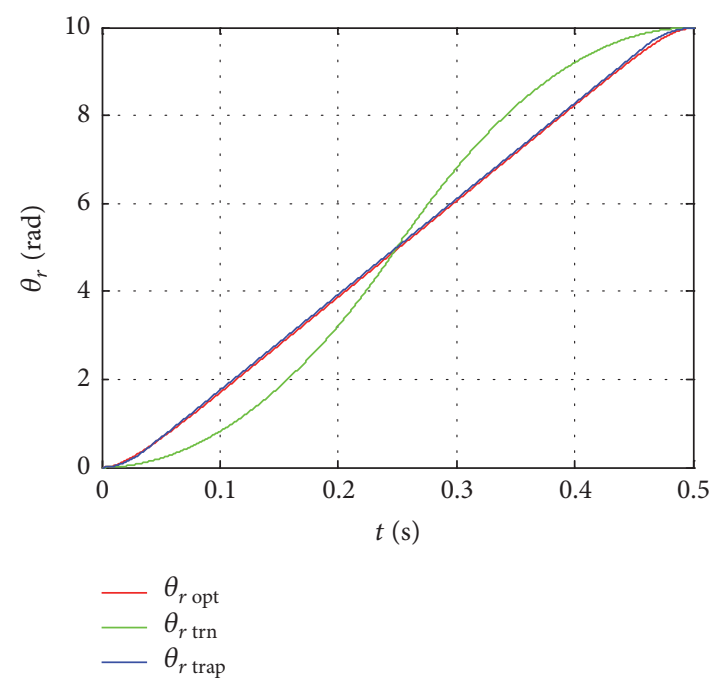

(b) Position profiles

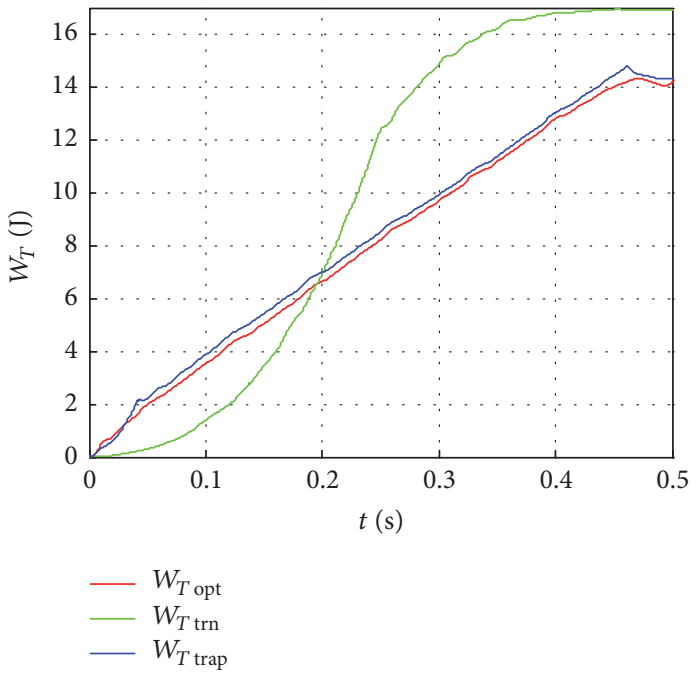

(d) Energy consumption

FIGURE 10: Experimental data of industrial drive $1.5 \mathrm{~kW}$ loaded torque containing constant and viscous component.

For both presented case studies the total energy expenditures of energy optimal control were lowest even if compared with energy near-optimal control strategy based on symmetrical trapezoidal speed profile. The differences in energy expenditures of these two control strategies are small, in the range of $1-3 \%$. Advantage of the proposed energy nearoptimal strategies was stressed in comparison with modified versions of energy near-optimal control (modified trapezoidal speed profile) and with position control based on triangular speed profile, the energy expenditures can be $40 \%$ higher.

The total energy loss of the near-optimal control strategy exploiting the trapezoidal speed profile over the optimal control might be reduced by using a cubic spline fit to four segments of the computed optimal position response, which is supposed as a further step of research as well as experimental verification.

\section{Appendix}

IM nominal parameters are as follows: $P_{N}=10 \mathrm{~kW}$, angular speed $\omega_{N}=117.6 \mathrm{rads}^{-1}$, current $I_{N}=32.4 \mathrm{~A}$, and torque $\Gamma_{N}=85 \mathrm{Nm}$. Resistances are $R_{s}=0.74 \Omega$ and $R_{r}=1 \Omega$, stator and rotor, respectively; inductances are $L_{s}=96.3 \mathrm{mH}$, $L_{m}=85.4 \mathrm{mH}$, and $L_{r}=96.1 \mathrm{mH}$, stator, mutual, and rotor, respectively; moment of inertia is $J_{r}=0.09 \mathrm{kgm}^{2}$ and polepairs number is $p=4$.

PMSM nominal parameters are as follows: $P_{N}=420 \mathrm{~kW}$, speed $\omega_{N}=125.67 \mathrm{rads}^{-1}$, current $I_{N}=630 \mathrm{~A}$, and torque $\Gamma_{N}=3342 \mathrm{Nm}$. Pole-pairs number is $p=3$, stator resistance, $R_{s}=0.0452 \Omega$, quadratic inductance, $L_{q}=5.7 \mathrm{mH}$, permanent mg. flux $\psi_{\mathrm{PM}}=1.2$ versus $c=3 p / 2$, and total moment of inertial, $J_{r}=470 \mathrm{kgm}^{2}$. Traction unit parameters are as follows: total unit mass $M_{u}=45,9 \mathrm{t}$ (consisting of 
one driven and one haulage carriage), wheel average diameter, $D_{w a}=0,91 \mathrm{~m}$, and gear ratio, $u=3,73$.

Parameters for IM equivalent circuit used in experiments are as follows: rated power, $P_{n}=1.5 \mathrm{~kW}$, at speed $n_{n}=$ $1420 \mathrm{rpm}$; rated current, $I_{n}=2.4 / 4.2 \mathrm{~A}, Y / \Delta$, and terminal voltage, $U=400 / 230, Y / \Delta$. Stator and rotor resistances are $R_{s}=5,155 \Omega$ and $R_{R}=4,426 \Omega$; stator and rotor inductances are $L_{s}=L_{\mathbf{R}}=0,291 \mathrm{H}$; mutual inductance is $L_{M}=0,271 \mathrm{H}$; moment of inertia is $J=0,0044 \mathrm{kgm}^{2}$.

\section{Nomenclature}

\section{Constant Parameters}

c: Dimensionless motor torque constant

$k_{P 1,2}$ : Precompensator gains

$L_{m}:$ Mutual inductance $[\mathrm{H}]$

$L_{s}:$ Stator inductance $[\mathrm{H}]$

$L_{r}:$ Rotor inductance $[\mathrm{H}]$

T: Matrix for transposition

$p$ : Number of pole pairs

$R: \quad$ Motor resistance $[\Omega]$

$R_{s}: \quad$ Stator resistance $[\Omega]$

$R_{r}$ : $\quad$ Rotor resistance related to stator $[\Omega]$

$a_{1}$ : Sum of stator and rotor resistances $[\Omega]$

$T_{r}: \quad$ Rotor time constant [s]

$c_{1-5}$ : Constants related to IM description

$J_{r}$ : $\quad$ Motor shaft related moment of inertia $\left[\mathrm{kgm}^{2}\right]$

$L_{d}: \quad$ PMSM inductance direct axis related $[\mathrm{H}]$

$L_{q}: \quad$ PMSM inductance quadrature axis related $[\mathrm{H}]$

$\mathbf{L}_{\mathbf{R}}$ : Matrix of reciprocal inductance $\left[\mathrm{H}^{-1}\right]$

$\Psi_{\text {PM: }}$ : Column vector of permanent magnet flux [Vs]

$k_{U}$ : Torque generator voltage constant $\left[\mathrm{Nms}^{-1} \mathrm{~V}^{-1}\right.$ ]

$k_{\omega}$ : Torque generator speed constant [Nm]

$k_{\gamma}$ : Torque generator torque constant $\left[\mathrm{s}^{-1}\right]$

$c \Psi:$ Motor torque constant $\left[\mathrm{NmA}^{-1}\right]$

$k_{T}$ : Motor torque constant $\left[\mathrm{NmA}^{-1}\right]$

$l_{1-4}$ : Eigenvalues of dynamical system matrix, $i=1,2,3,4$

A: Coefficient of constant friction [Nm]

$B$ : Coefficient of viscous friction [Nms]

C: Coefficient of quadratic friction [Nms ${ }^{2}$ ]

$T_{m}$ : Time of maneuver (from rest to rest) $[\mathrm{s}]$
$T_{0}$ : Interval of constant cruising speed [s]

$\lambda_{i}: \quad$ Lagrange multipliers, $i=1,2,3$

$\theta_{r d}$ : Position demanded value [rad]

$\omega_{r d}: \quad$ Rotor speed demanded value $\left[\mathrm{rads}^{-1}\right]$,

$\varepsilon_{p}: \quad$ Peak acceleration and deceleration $\left[\mathrm{rads}^{-2}\right]$

$k_{i}$ : Constants for motor consumption

description, $i=1,2,3,4,5$

$\kappa$ : $\quad$ Ratio between acceleration time, $T_{\varepsilon}$ and time of maneuver, $T_{m}$

$c_{m}: \quad$ Dimensionless motor constant

$i_{d \mathrm{dem}}$ : Demanded value of excitation current in $d$ axis of PMSM and IM

$\varepsilon_{\text {p opt }}:$ Optimal acceleration $\left[\mathrm{rads}^{-2}\right.$ ]

$\omega_{p}: \quad$ Cruising speed $\left[\mathrm{rads}^{-1}\right]$,

$T_{\omega}$ : Time constant of speed control [s]

$T_{s \theta}: \quad$ Position settling time $[\mathrm{s}]$

$n$ : Order of the dynamical system

$P_{i}$ : Coefficient for control system stability evaluation, $i=1,2,3$

$D_{w a}:$ Average diameter of driving wheel [m]

$u$ : $\quad$ Gear ratio between motor and wheel

$m_{t}$ : Mass of train [t]

$l_{\mathrm{td}}: \quad$ Demanded travelled distance $[\mathrm{m}]$

$m$ : Number of traction motors of vehicle.

Variables

$t: \quad$ Time [s]

U: $\quad$ Phasor of stator voltages [V]

I: $\quad$ Phasor of stator currents [A]

$\Psi$ : $\quad$ Phasors of stator mg. flux of PMSM and rotor mg. flux of IM, respectively, [Vs]

$\mathbf{P}\left(\omega_{r}\right): \quad$ System matrix for IM

$\Gamma_{\mathrm{el}}$ : $\quad$ Electrical torque (matrix form)

[Nm]

$\Gamma_{L}: \quad$ Load torque (matrix form) $[\mathrm{Nm}]$

$\mathbf{P}\left(\omega_{r}, R_{s}\right)$ : System matrix of PMSM

$\varepsilon_{r}: \quad$ Rotor angular acceleration $\left[\mathrm{rads}^{-2}\right]$

$\omega_{r}: \quad$ Rotor angular speed $\left[\mathrm{rads}^{-1}\right]$

$\theta_{r}: \quad$ Rotor position [rad]

$u_{d}: \quad$ Excitation voltage of $d$ axes [V]

$u_{q}: \quad$ Excitation voltage of $q$ axes [V]

$i_{d}$ : $\quad$ Direct component of stator current [A]

$i_{q}: \quad$ Quadratic component of stator

current [A]

$i_{d \mathrm{dem}}: \quad$ Demanded value of direct current

$i_{q \text { dem }}$ : $\quad$ Demanded value of quadratic current [A]

$\gamma_{e}: \quad$ Motor electrical torque [Nm]

$\gamma_{L}$ : Load torque (as a function of $\omega_{r}$ )

$I_{T}: \quad$ Functional for energy

minimization $[\mathrm{J}]$

$I_{P}: \quad$ Cost function for energy minimization 
$T_{\varepsilon}: \quad$ Acceleration and deceleration time [s]

$T_{\varepsilon \text { opt }}$ : Optimal value of acceleration time [s]

$\varepsilon_{\text {popt }}:$ Optimal acceleration $\left[\mathrm{rads}^{-2}\right]$

$W_{T}:$ Motor total energy consumption [J]

$V_{V}$ : Vehicle speed $[\mathrm{km} / \mathrm{h}]$

$F_{t}$ : Traction force [N]

$F_{p}: \quad$ Force to cover passive resistances [N]

$F_{s}: \quad$ Force to cover slope resistances $[\mathrm{N}]$

$F_{b}$ : Braking force [N]

$p_{0}: \quad$ Passive traction resistance $[\mathrm{N} / \mathrm{kN}]$

$l_{t}: \quad$ Travelled distance $[\mathrm{m}]$

$W_{T}$ : Vehicle total energy consumption [Wh].

\section{Subscripts}

$s: \quad$ Related to stator

$r$ : $\quad$ Related to rotor

$i$ : Ideal, related to precomputed

variables

$m$ : Related to mutual inductance

$N$ : $\quad$ Related to rated parameters

sl: $\quad$ Slip angular speed

$d$ : $\quad$ Related to direct axis

$q$ : $\quad$ Related to quadrature axes

opt: Related to energy optimal

trap: Related to trapezoidal speed profile

trn: Related to triangular speed profile

curr: Related to defined value of current.

\section{Competing Interests}

The authors declare that there is no conflict of interests regarding the publication of this paper.

\section{Acknowledgments}

The authors wish to thank the Slovak Grant Agency VEGA for support of research Grant 1/0794/14.

\section{References}

[1] J. Wang, "Optimal control and energy efficiency_integration of design and engineering," in Proceedings of the 18th International Conference on Automation and Computing (ICAC '12), pp. 1-6, Loughborough, UK, 2012.

[2] L. S. Pontryagin, V. G. Boltyanskij, R. V. Gamkrelidze, and F. Mishchenko, The Mathematical Theory of Optimal Processes, Pergamon Press, 1964.

[3] D. W. Novotny and T. A. Lipo, Vector Control and Dynamics of AC Drives, Clarendon Press, Oxford, UK, 1996.

[4] S. J. Dodds, Feedback Control: Linear, Nonlinear and Robust Techniques and Design with Industrial Applications, Springer, London, UK, 2015.

[5] Y. Wang, B. Ning, F. Cao, B. De Schutter, and T. J. J. Van Den Boom, "A survey on optimal trajectory planning for train operations," in Proceedings of the IEEE International Conference on Service Operations, Logistics and Informatics (SOLI '11), pp. 589-594, Beijing, China, July 2011.
[6] T. H. Pham, I. Prodan, D. Genon-Catalot, and L. Lefevre, "Efficient energy management for an elevator system under a constrained optimization framework, integration of design and engineering," in Proceedings of the 19th International Conference on System Theory, Control and Computing, pp. 613-618, Cheile Gradistei, Romania, 2015.

[7] F. Blank, T. Roser, and J. Roth-Stielow, "Drive system loss minimizing trajectories using constrained non-linear optimization," in Proceedings of the 11th IEEE International Conference on Power Electronics and Drive Systems (PEDS '15), pp. 986-991, IEEE, Sydney, Australia, June 2015.

[8] Z. Xuejun, T. Izumi, and Z. Yiting, "Energy-optimal planning of a driving system with coulomb and viscous frictions," in Proceedings of the IEEE International Conference on Mechatronics and Automation (ICMA '06), pp. 623-628, IEEE, Luoyang, China, June 2006.

[9] C. H. Kim and B. K. Kim, "Energy-saving 3-step velocity control algorithm for battery-powered wheeled mobile robots," in Proceedings of the IEEE International Conference on Robotics and Automation (ICRA '05), pp. 2375-2380, IEEE, Barcelona, Spain, April 2005.

[10] S. Thirachai, P. Komeswarakul, U. Supakchukul, and J. Suwatthikul, "Trapezoidal velocity trajectory generator with speed override capability," in Proceedings of the International Conference on Control, Automation and Systems (ICCAS '10), pp. 1468-1472, Geonggi-do, Korea, October 2010.

[11] Y. H. Luo and H. Z. Xu, "Direct robust adaptive control of high speed train based on nonlinear and time-varying models," International Journal of Control and Automation, vol. 6, no. 4, pp. 397-410, 2013.

[12] D. Lu, M. Ouyang, J. Gu, and J. Li, "Optimal velocity control for a battery electric vehicle driven by permanent magnet synchronous motors," Mathematical Problems in Engineering, vol. 2014, Article ID 193960, 14 pages, 2014.

[13] A. K. Yadav, P. Gaur, S. K. Jha, J. R. P. Gupta, and A. P. Mittal, "Optimal speed control of hybrid electric vehicles," Journal of Power Electronics, vol. 11, no. 4, pp. 393-400, 2011.

[14] V. De Martinis and M. Gallo, "Models and methods to optimise train speed profiles with and without energy recovery systems: a suburban test case," Procedia-Social and Behavioral Sciences, vol. 87, pp. 222-233, 2013.

[15] G. M. Sheepmaker and M. P. R. Goverde, "Effect of Regenerative braking on energy-efficient train control," in Proceedings of the International Conference on Advanced Systems in Public Transport, Rotterdam, The Netherlands, 2015.

[16] A. R. Albrecht, P. G. Howlett, P. J. Pudney, and X. Vu, "Energy-efficient train control: from local convexity to global optimization and uniqueness," Automatica, vol. 49, no. 10, pp. 30723078, 2013.

[17] E. Khmelnitsky, "On an optimal control problem of train operation," IEEE Transactions on Automatic Control, vol. 45, no. 7, pp. 1257-1266, 2000.

[18] J. Vittek and S. J. Dodds, Forced Dynamics Control of Electric Drives, EDIS Publishing Centre of Zilina University, Zilina, Slovakia, 2003, http://www.kves.uniza.sk/?menu=proj\&page= Elearn.

[19] B. K. Bose, Modern Power Electronics and A.C. Drives, Prentice Hall, PTR, 2002.

[20] P. Fedor and D. Perdukova, "Energy optimization of a dynamic system controller," in Proceedings of the International Joint Conference on Advances in Intelligent Systems and Computing AISC CISIS '12-ICEUTE '12-SOCO '12 Special Session, 
vol. 189, pp. 361-369, 2013, http://link.springer.com/chapter/ 10.1007/978-3-642-33018-6_37.

[21] J. Vittek, P. Bris, M. Pospisil, P. Butko, and T. Fedor, "Energy saving position control of PMSM drives with constant, linear and quadratic torques," in Proceedings of the IEEE International Conference on Power Electronics, Drives and Energy Systems (PEDES '14), Mumbai, India, December 2014.

[22] V. Šlapák, K. Kyslan, M. Lacko, V. Fedák, and F. Ďurovský, "Finite control set model predictive speed control of a DC motor," Mathematical Problems in Engineering, vol. 2016, Article ID 9571972, 10 pages, 2016.

[23] J. Boldea and A. S. Nasar, Vector Control of AC Drives, CRC Press, London, UK, 1992.

[24] P. Brandstetter and T. Krecek, "Speed and current control of permanent magnet synchronous motor drive using IMC controllers," Advances in Electrical and Computer Engineering, vol. 12, no. 4, pp. 3-10, 2012.

[25] Y. Rozanov, S. Ryvkin, E. Chaplygin, and P. Voronin, Power Electronics-Basics, Operating Principles, Design, Formulas and Applications, CRC Press, London, UK, 2016.

[26] S. J. Dodds, "Settling time formulae for the design of control systems with linear closed loop dynamics," in Proceedings of the International Conference AC\&T, University of East London, London, UK, 2007.

[27] J. Vittek, P. Butko, J. Ftorek, and P. Fedor, "Case study of energy optimal and energy near-optimal control algorithms for the drives with constant, linear and quadratic frictions," in Proceedings of the 14th International Conference IEEE PEMC, pp. 664-671, Varna, Bulgaria, 2016. 


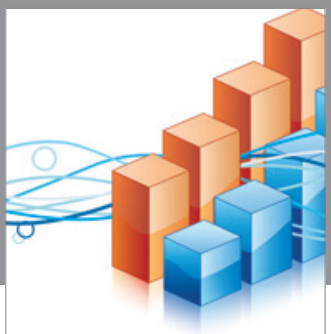

Advances in

Operations Research

vatem alat4

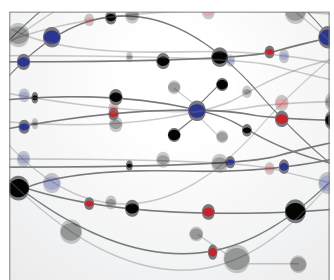

\section{The Scientific} World Journal
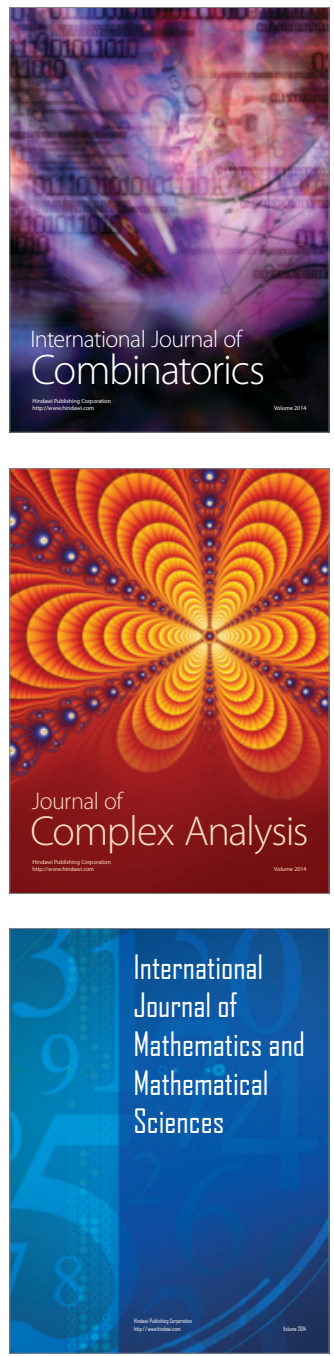
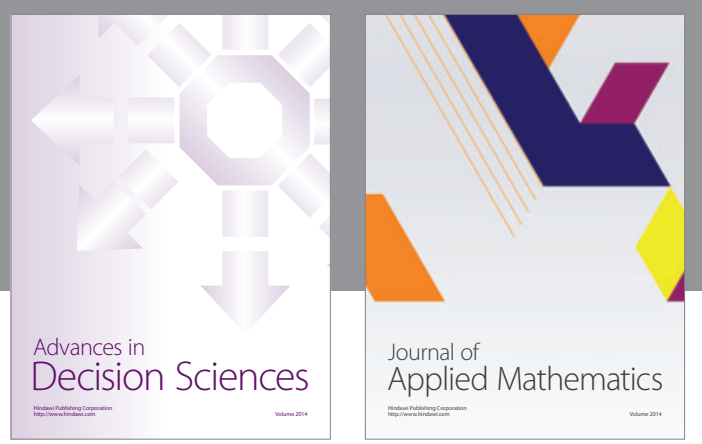

Algebra

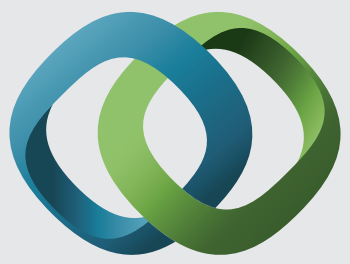

\section{Hindawi}

Submit your manuscripts at

https://www.hindawi.com
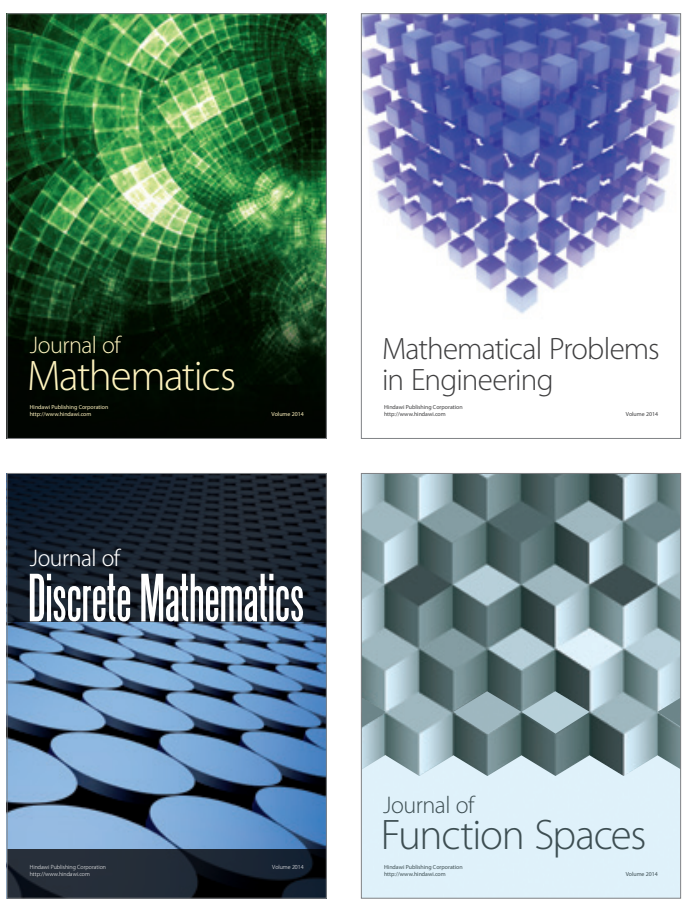

Mathematical Problems in Engineering
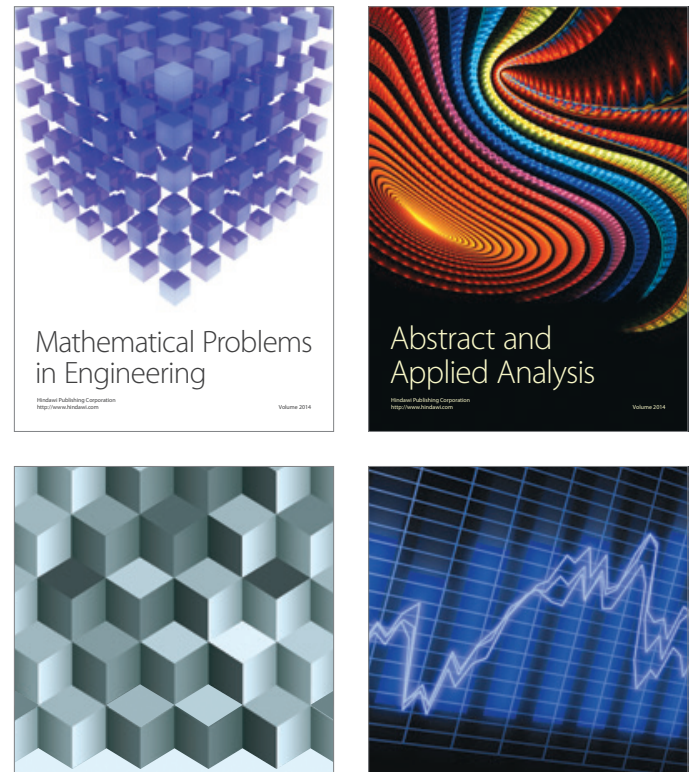

Journal of

Function Spaces

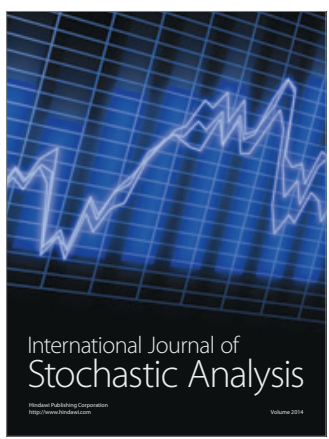

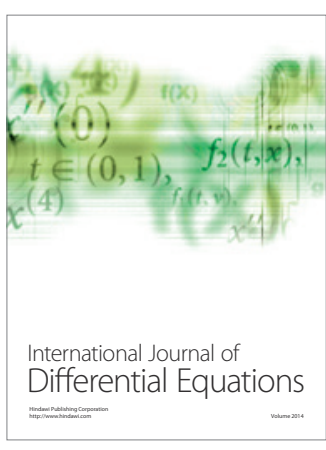
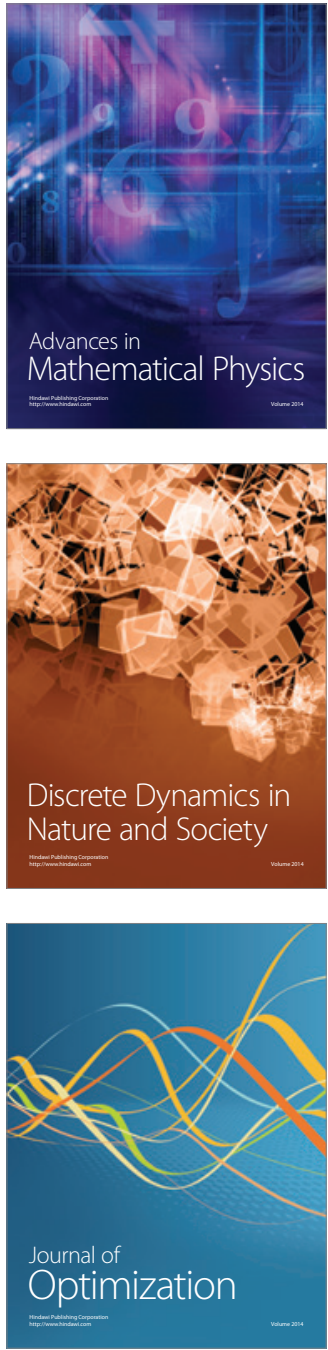